\title{
Mapping dark matter on the celestial sphere with weak gravitational lensing
}

\author{
Christopher G. R. Wallis ${ }^{\circledR},{ }^{1}$ Matthew A. Price, ${ }^{1 \star}$ Jason D. McEwen, ${ }^{1}$ Thomas D. Kitching, ${ }^{1}$ \\ Boris Leistedt ${ }^{2} \dagger$ and Antoine Plouviez ${ }^{1}$ \\ ${ }^{1}$ Mullard Space Science Laboratory, University College London, Surrey RH5 6NT, UK \\ ${ }^{2}$ Center for Cosmology and Particle Physics, Department of Physics, New York University, New York, NY 10003, USA
}

Accepted 2021 November 3. Received 2021 October 27; in original form 2021 July 21

\begin{abstract}
Convergence maps of the integrated matter distribution are a key science result from weak gravitational lensing surveys. To date, recovering convergence maps has been performed using a planar approximation of the celestial sphere. However, with the increasing area of sky covered by dark energy experiments, such as Euclid, the Vera Rubin Observatory's Legacy Survey of Space and Time (LSST), and the Nancy Grace Roman Space Telescope, this assumption will no longer be valid. We recover convergence fields on the celestial sphere using an extension of the Kaiser-Squires estimator to the spherical setting. Through simulations, we study the error introduced by planar approximations. Moreover, we examine how best to recover convergence maps in the planar setting, considering a variety of different projections and defining the local rotations that are required when projecting spin fields such as cosmic shear. For the sky coverages typical of future surveys, errors introduced by projection effects can be of the order of tens of percent, exceeding 50 per cent in some cases. The stereographic projection, which is conformal and so preserves local angles, is the most effective planar projection. In any case, these errors can be avoided entirely by recovering convergence fields directly on the celestial sphere. We apply the spherical Kaiser-Squires mass-mapping method presented to the public Dark Energy Survey science verification data to recover convergence maps directly on the celestial sphere.
\end{abstract}

Key words: methods: data analysis - cosmology: observations.

\section{INTRODUCTION}

Weak gravitational lensing distorts the shape and size of images of distant galaxies due to the gravitational influence of matter perturbations along the line of sight (see e.g. Bartelmann \& Schneider 2001; Schneider 2005; Munshi et al. 2008; Heavens 2009). The amplitude of the distortion - a change in the ellipticity (third flattening or third eccentricity) and apparent size of an object - contains information on the integrated Newtonian potential and can be used to estimate the integrated mass distribution. The lensing effect is dependent on the total mass distribution and therefore, because massive structures are dominated by dark matter, the mass distributions recovered by weak lensing are colloquially referred to as mass-maps of the dark matter of the Universe. The creation of such maps constitutes one of the main empirical observations that underpins the dark matter paradigm (Clowe et al. 2006).

The most common approach to extract cosmological information from weak lensing surveys is to compute the two-point correlation function (e.g. Kilbinger 2015) or power spectrum (e.g. Alsing et al. 2016) from observational data and compare to an expectation from theory. However, such analyses are sensitive only to the Gaussian component of the underlying field. To capture the entire information

\footnotetext{
^E-mail: m.price.17@ucl.ac.uk
}

$\dagger$ NASA Einstein Fellow content of the shear field, higher order statistics (e.g. Munshi et al. 2011) or phase information (e.g. Coles \& Chiang 2000) must be considered. Recovering mass-maps provides the basis for performing a wide variety of complimentary higher order statistical analyses that probe the non-Gaussian structure of the dark matter distribution. For example, properties of dark matter can then be studied using analyses based on peak and void statistics (e.g. Lin \& Kilbinger 2015a,b; Lin, Kilbinger \& Pires 2016; Peel et al. 2016), Minkowski functions (e.g. Kratochvil et al. 2012; Munshi et al. 2012; Petri et al. 2013), or wavelets (cf. Hobson, Jones \& Lasenby 1998; Aghanim et al. 2003; Vielva et al. 2004, McEwen et al. 2005), to name just a few.

Further to this, mass-mapping provides an efficient way to crosscorrelate weak lensing data with other cosmological data (e.g. with observations of the cosmic microwave background; Liu \& Hill 2015). More directly, dark matter maps are of interest for galaxy evolution studies: it is known from simulations that the dark matter structure should exhibit a filamentary or 'cosmic web' structure inference of which can then provide dark matter environmental information that can then be used in galaxy evolution studies (Brouwer et al. 2016). Finally, mass-mapping is a continuation of cartography on to the cosmic scale - the making of such maps is therefore laudable in its own right.

Recovering mass-maps requires solving an inverse problem to recover the underlying mass distribution from the observable cosmic shear. There are a number of approaches to estimating mass-maps from weak lensing data. The method most commonly used on 
large scales is colloquially known as 'Kaiser-Squires' (KS) and is named after the paper in which the method was first described (Kaiser \& Squires 1993). This approach is based on a direct Fourier inversion of the equations relating the observed shear field to the convergence field, which is a scaled version of the integrated mass distribution. Although it is widely known that such an approach, based on a direct Fourier inversion, is not robust to noise, the method remains in widespread use today (in practice, the resultant mass-map is smoothed to mitigate noise). Indeed, the KS method has been used to recover mass-maps from data from by a number of recent weak lensing surveys, including data from the Cosmic Evolution Survey (COSMOS; Scoville et al. 2007), the CanadaFrance-Hawaii Telescope Lensing Survey (CFHTLenS; Heymans et al. 2012), and the Dark Energy Survey (DES; Flaugher et al. 2015) Science Verification (SV) data (respectively, Massey et al. 2007; Van Waerbeke et al. 2013; Chang et al. 2015). Alternative massmapping techniques to recover the convergence field have also been developed, however, these are not typically in widespread use and in many cased are focused on the galaxy cluster scale. On the galaxy cluster scale, parametric models (e.g. Jullo et al. 2007) and nonparametric methods (e.g. Massey et al. 2015; Lanusse et al. 2016; Price et al. 2021) have been considered. Szepietowski et al. (2014) have investigated the use of phase information from galaxy number counts to improve the reconstruction.

While the methods discussed above focus on recovering the twodimensional convergence field, which represents the integrated mass distribution along the line of sight, it is also possible to recover the full three-dimensional gravitational potential. Such an approach involves an additional inverse problem and thus an additional level of complexity. This has been considered by a number of works (Bacon \& Taylor 2003; Massey et al. 2004; Taylor et al. 2004; Simon, Taylor \& Hartlap 2009; VanderPlas et al. 2011; Leonard, Dupé \& Starck 2012; Simon 2013; Leonard, Lanusse \& Starck 2014)

In general, mass-mapping techniques for weak lensing consider a small field-of-view of the celestial sphere, which is approximated by a tangent plane. The mass-mapping formalism is then developed in a planar setting, where a planar two-dimensional Fourier transform is adopted. Such an assumption will not be appropriate for forthcoming surveys, which will observe significant fractions of the celestial sphere, such as the Kilo Degree Survey (KiDS ${ }^{1}$; de Jong et al. 2013), DES ${ }^{2}$ (Flaugher et al. 2015), Euclid ${ }^{3}$ (Laureijs et al. 2011), $\mathrm{LSST}^{4}$ (LSST Science Collaboration et al. 2009), and the Nancy Grace Roman Space Telescope ${ }^{5}$ (Spergel et al. 2015). Fig. 1 illustrates the approximate sky coverage for DES SV data, DES full data, and Euclid observations, from which it is apparent that planar approximations will become increasingly inaccurate as sky coverage areas grow over time. Existing mass-mapping techniques that are based on planar approximations therefore cannot be directly applied to forthcoming observations, without introducing significant errors into subsequently inferred statistics (see e.g. Vallis, Wallis \& Kitching 2018, for an analysis of projection effects on peak statistics and minkowski functionals). This work aims to highlight the necessity of spherical methods, by demonstrating the inevitability of errors introduced by planar projections, and does not attempt to quantify the effect of such projection errors on global statistics.

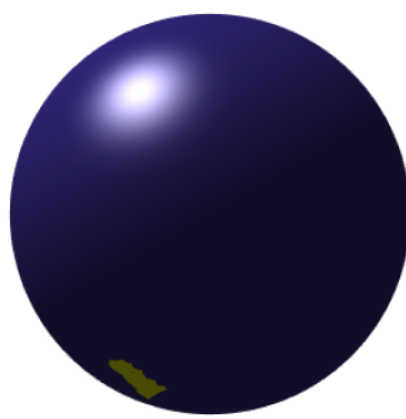

(a) DES SV

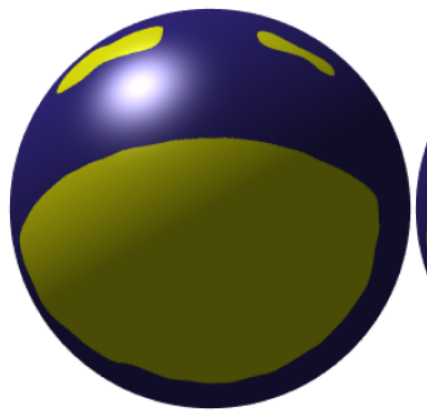

(c) Euclid (front and rear views)

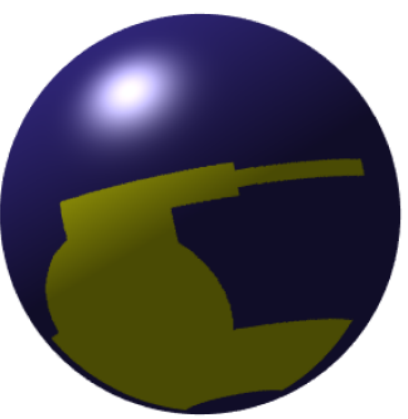

(b) DES full

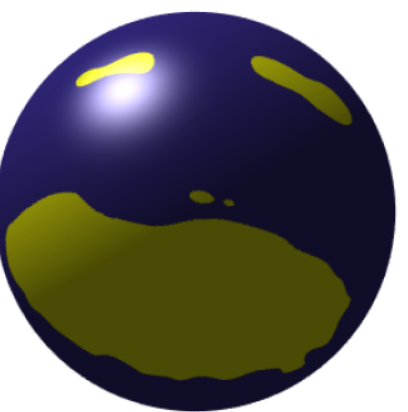

Figure 1. Approximate coverage area of different weak lensing surveys illustrated on the celestial sphere. In particular, the coverage area corresponding to DES SV observations, DES full observations, and Euclid observations are shown. It is apparent that existing planar mass-mapping techniques will not be appropriate for the large coverage areas of forthcoming surveys. We extend the KS technique for mass-mapping to the spherical setting in this article, in order to recover mass-maps on the celestial sphere.

In this article, we consider the KS approach for recovering massmaps defined on the full celestial sphere. While the harmonic space expressions in the spherical setting relating the observed shear field to the convergence field, via the lensing potential, have been presented already (e.g. Taylor 2001; Castro, Heavens \& Kitching 2005; Pichon et al. 2010), to the best of our knowledge, naive Fourier inversion on the celesital sphere (i.e. spherical KS) has not been considered previously. We compare the spherical KS formalism with the planar case, considering several different spherical projections. ${ }^{6}$ Spherical mass-mapping techniques have also been considered by Pichon et al. (2010), where a maximum a posteriori (MAP) estimator was presented. In addition, the authors consider using a Wiener filter to denoise the shear in advance of attempting to recover convergence maps. However, as far as we are aware, these techniques have not been applied to observational data. The spherical KS technique that we present here is a first step towards more sophisticated spherical massmapping techniques that will be the focus of future work. In practice, only partial-fields defined on the celestial sphere are observed. The KS estimator suffers due to leakage induced by the masking of the observed region (it is well-known that the decomposition of a spin field into scalar and pseudo-scalar components, and consequently mass-mapping, is not unique on a manifold with boundary; Bunn

\footnotetext{
${ }^{1}$ http://kids.strw.leidenuniv.nl

${ }^{2}$ http://www.darkenergysurvey.org

${ }^{3}$ http://euclid-ec.org

${ }^{4}$ https://www.lsst.org

${ }^{5}$ https://roman.gsfc.nasa.gov
}

${ }^{6}$ An alternative to recovering mass-maps directly on the sphere is to tile the
celestial sphere and perform mass-mapping on planar patches, as considered
for the lensing of the cosmic microwave background by Plaszczynski et al.
(2012). An extension of this work to galaxy lensing, when shear is observed,
would be of great interest. 
et al. 2003). Pure mode estimators on the celestial sphere can be developed to remove this leakage (e.g. Leistedt et al. 2017). Furthermore, the impact of noise can be mitigated by the use of regularization methods adapted to the spherical setting (e.g. Wallis, Wiaux \& McEwen 2016).

The remainder of this article is structured as follows. In Section 2, we briefly review the mathematical background of spin fields on the sphere and weak gravitational lensing. Mass-mapping on the celestial sphere is presented in Section 3. In Section 4, we use simulations to compare the spherical case to a variety of planar settings for various spherical projections. In Section 5, we present an application of the spherical KS technique to DES SV data in order to recover spherical mass-maps. Concluding remarks are made in Section 6. Throughout, we adopt the cubehelix (Green 2011) colour scheme.

\section{BACKGROUND}

Weak gravitational lensing gives rise to scalar and spin fields defined on the celestial sphere. For example, the observed shear field induced by weak gravitational lensing is a spin \pm 2 field. We therefore review scalar and spin fields on the sphere and their harmonic representation, before reviewing the mathematical details of rotation and the Dirac delta function on the sphere, which we make use of subsequently when considering mass-mapping on the celestial sphere. Weak gravitational lensing in the three-dimensional spherical setting is then reviewed concisely.

\subsection{Spin fields on the sphere}

Square integrable spin fields on the $\operatorname{sphere}_{s} f$, with integer spin $s \in$ $\mathbb{Z}$, are defined by their behaviour under local rotations. By definition, a spin field transforms as

${ }_{s} f^{\prime}(\omega)=\mathrm{e}^{-\mathrm{i} x \chi}{ }_{s} f(\omega)$,

under a local rotation by $\chi \in[0,2 \pi)$, where the prime denotes the rotated field (Newman \& Penrose 1966; Goldberg et al. 1967; Kamionkowski, Kosowsky \& Stebbins 1997; Zaldarriaga \& Seljak 1997). ${ }^{7}$ It is important to note that the rotation considered here is not a global rotation on the sphere but rather a rotation by $\chi$ in the tangent plane centred on the spherical coordinates $\omega=(\theta, \varphi) \in \mathbb{S}^{2}$, with co-latitude $\theta \in[0, \pi]$ and longitude $\varphi \in[0,2 \pi)$. The case $s=0$ reduces to the standard scalar setting.

The canonical basis for scalar fields defined on the sphere are given by the (scalar) spherical harmonics $Y_{\ell m}$. Basis functions for spin fields can be defined by applying spin lowering and raising operators to the scalar spherical harmonics. Spin raising and lowering operators, $\partial$ and $\bar{\partial}$ respectively, increment and decrement the spin order of a spin- $s$ field by unity and are defined by

$\partial \equiv-\sin ^{s} \theta\left(\frac{\partial}{\partial \theta}+\frac{\mathrm{i}}{\sin \theta} \frac{\partial}{\partial \varphi}\right) \sin ^{-s} \theta$

and

$\bar{\delta} \equiv-\sin ^{-s} \theta\left(\frac{\partial}{\partial \theta}-\frac{\mathrm{i}}{\sin \theta} \frac{\partial}{\partial \varphi}\right) \sin ^{s} \theta$,

\footnotetext{
${ }^{7}$ The sign convention adopted for the argument of the complex exponential differs to the original definition (Newman \& Penrose 1966) but is identical to the convention used typically in astrophysics (Kamionkowski et al. 1997; Zaldarriaga \& Seljak 1997).
}

respectively (Newman \& Penrose 1966; Goldberg et al. 1967; Kamionkowski et al. 1997; Zaldarriaga \& Seljak 1997). When applied to spherical harmonics, the spin raising and lowering operators take the form

$\partial_{s} Y_{\ell m}(\omega)=[(\ell-s)(\ell+s+1)]^{1 / 2}{ }_{s+1} Y_{\ell m}(\omega)$

and

$\bar{ळ}_{s} Y_{\ell m}(\omega)=-[(\ell+s)(\ell-s+1)]^{1 / 2}{ }_{s-1} Y_{\ell m}(\omega)$,

respectively (see e.g. Zaldarriaga \& Seljak 1997). The spin-s spherical harmonics can, thus, be expressed in terms of the scalar (spinzero) harmonics through the spin, raising and lowering operators by

${ }_{s} Y_{\ell m}(\omega)=\left[\frac{(\ell-s) !}{(\ell+s) !}\right]^{1 / 2} \partial^{s} Y_{\ell m}(\omega)$,

for $0 \leq s \leq \ell$, and by

${ }_{s} Y_{\ell m}(\omega)=(-1)^{s}\left[\frac{(\ell+s) !}{(\ell-s) !}\right]^{1 / 2} \bar{\partial}^{-s} Y_{\ell m}(\omega)$,

for $-\ell \leq s \leq 0$, where $Y_{\ell m}$ denote the scalar (spin-zero) spherical harmonics.

Due to the orthogonality and completeness of the spin spherical harmonics, a spin field on the sphere can be decomposed into its harmonic representation by

${ }_{s} f(\omega)=\sum_{\ell=0}^{\infty} \sum_{m=-\ell}^{\ell} \hat{f}_{\ell m s} Y_{\ell m}(\omega)$.

The harmonic coefficients of ${ }_{s} f$, denoted by ${ }_{s} \hat{f}$, are given by the usual projection on to the basis functions

${ }_{s} \hat{f}_{\ell m}=\left\langle{ }_{s} f, s Y_{\ell m}\right\rangle=\int_{\mathbb{S}^{2}} \mathrm{~d} \Omega(\omega)_{s} f(\omega)_{s} Y_{\ell m}^{*}(\omega)$,

where the rotation invariant measure on the sphere is given by $\mathrm{d} \Omega(\omega)=\sin \theta \mathrm{d} \theta \mathrm{d} \varphi$, the inner product on the sphere is denoted by $\langle\cdot, \cdot\rangle$ and $\cdot^{*}$ denotes complex conjugation. In practice, we consider harmonic coefficients up to a maximum degree $\ell_{\max }$, i.e. signals on the sphere band-limited at $\ell_{\max }$ with $f_{\ell m}=0, \forall \ell \geq \ell_{\max }$, in which case, summations over $\ell$ can be truncated at $\ell_{\max }$. For notational brevity, we sometimes do not explicitly show the limits of summation where these can be inferred easily.

\subsection{Rotation on the sphere}

We subsequently consider the rotation of fields on the sphere, defined by application of the rotation operator $\mathcal{R}_{\rho}$, where the rotation is parametrized by the Euler angles $\rho=(\alpha, \beta, \gamma) \in \mathrm{SO}(3)$. We adopt the $z y z$ Euler convention corresponding to the rotation of a physical body in a fixed coordinate system about the $z, y$, and $z$ axes by $\gamma$, $\beta$, and $\alpha$, respectively. Often, we consider rotations with $\gamma=0$ and adopt the shorthand notation $\mathcal{R}_{\omega}=\mathcal{R}_{(\varphi, \theta, 0)}$.

The spin spherical harmonic functions are rotated by (e.g. McEwen et al. 2015)

$\left(\mathcal{R}_{\rho} s Y_{\ell m}\right)(\omega)=\sum_{n=-\ell}^{\ell} D_{n m}^{\ell}(\rho)_{s} Y_{\ell n}(\omega)$,

where $D_{n m}^{\ell}$ are the Wigner $D$-functions (Varshalovich, Moskalev \& Khersonskii 1989), which follows from the additive property of the Wigner $D$-functions (Marinucci \& Peccati 2011). 
The Wigner $D$-functions may also be related to the spin spherical harmonics by (Goldberg et al. 1967)

$\mathrm{e}^{-\mathrm{i} s \gamma}{ }_{s} Y_{\ell m}(\beta, \gamma)=(-1)^{s} \sqrt{\frac{2 \ell+1}{4 \pi}} D_{m,-s}^{\ell *}(\alpha, \beta, \gamma)$.

\subsection{Dirac delta on the sphere}

We subsequently make use of the Dirac delta function on the sphere $\delta^{\mathrm{D}}$, defined by

$$
\begin{aligned}
& \left(\mathcal{R}_{\omega^{\prime}} \delta^{\mathrm{D}}\right)(\omega)=\frac{1}{\sin \theta} \delta^{1 \mathrm{D}}\left(\theta-\theta^{\prime}\right) \delta^{1 \mathrm{D}}\left(\varphi-\varphi^{\prime}\right) \\
& =\sum_{\ell=0}^{\infty} \sum_{m=-\ell}^{\ell} Y_{\ell m}^{*}\left(\omega^{\prime}\right) Y_{\ell m}(\omega),
\end{aligned}
$$

where $\delta^{1 \mathrm{D}}(\cdot)$ denotes the standard one-dimensional (Euclidean) Dirac delta. The spherical harmonic coefficients of the Dirac delta defined on the sphere are given by

$\hat{\delta}_{\ell m}^{\mathrm{D}}=Y_{\ell m}^{*}(\mathbf{0})=\sqrt{\frac{2 \ell+1}{4 \pi}} \delta_{m 0}$.

\subsection{Weak gravitational lensing}

We now turn our attention to weak gravitational lensing, concisely reviewing the related mathematical background, which is covered in more depth in several review articles (e.g. Bartelmann \& Schneider 2001; Schneider 2005; Munshi et al. 2008; Heavens 2009).

The weak gravitational lensing effect is typically expressed in terms of the lensing potential $\phi$, which depends on the integrated deflection angle along the line of sight, sourced by the local Newtonian potential $\Phi$

$\phi(r, \omega)=\frac{2}{c^{2}} \int_{0}^{r} \mathrm{~d} r^{\prime} \frac{f_{K}\left(r-r^{\prime}\right)}{f_{K}(r) f_{K}\left(r^{\prime}\right)} \Phi\left(r^{\prime}, \omega\right)$,

where $c$ is the speed of light in a vacuum, $r$ and $r^{\prime}$ are comoving distances, and $\omega=(\theta, \varphi)$ denote spherical coordinates, as defined previously. The angular diameter distance factor reads

$f_{K}(r)=\left\{\begin{array}{ll}\sin (r), & \text { if } K=1 \\ r, & \text { if } K=0 \\ \sinh (r), & \text { if } K=-1\end{array}\right.$,

for cosmologies with positive $(K=1)$, flat $(K=0)$, and negative $(K=-1)$ global curvatures. This expression assumes the Born approximation. The gravitational potential is related to the density field by Poisson's equation

$\nabla^{2} \Phi(r, \omega)=\frac{3 \Omega_{\mathrm{M}} H_{0}^{2}}{2 a(r)} \delta(r, \omega)$,

where $\Omega_{\mathrm{M}}$ is the current average matter density of the Universe as a fraction of the critical density, $H_{0}$ is the current expansion rate of the Universe, $a(r)$ is the scale factor, and $\delta$ is the fractional matter overdensity. Equations (15) and (17) relate the matter perturbations $\delta$ to the lensing potential $\phi$.

The lensing potential describes how light from a background source (e.g. galaxy) at a position $(r, \omega)$ is distorted by the lensing effect. This deflection, to first order, affects the images of galaxies in two ways. First, images of background sources are magnified by the convergence $\kappa$, which is related to the lensing potential by

$0 \kappa(r, \omega)=\frac{1}{4}(\check{\partial} \bar{\partial}+\bar{\varnothing} \check{\partial}) 0 \phi(r, \omega)$, through the spin raising and lowering operators introduced in equations (2) and (3). The convergence is not measured directly in weak lensing experiments because the intrinsic magnitude of galaxy sizes is unknown. Here and subsequently, we denote the spin of each field explicit with a proceeding subscript, i.e. $0 \phi=\phi$ and $0 \kappa=\kappa$ are both spin-zero (scalar) fields. Secondly, images of background sources are sheared by $2 \gamma$, which is related to the lensing potential by

$2 \gamma(r, \omega)=\frac{1}{2} \partial \partial 0 \phi(r, \omega)$,

where we make it explicit that the shear is a spin-2 field. Upon averaging the shapes of many galaxies, one would expect the intrinsic shear to average to zero (i.e. there is no preferred orientation). Hence, one can measure shear by averaging the shapes of many galaxies. In the remainder of this article, we do not consider 'tomography' (the separation of a source galaxy sample into populations labelled by redshift or time) and so drop the radial dependence shown in the above equations (for notational brevity, henceforth, we typically do not show the angular dependence either). For further information, see the discussions in Kitching et al. (2016) on spherical-radial and spherical-Bessel representations of the shear field.

In general, the potential $0 \phi$ can be decomposed into its parity even and parity odd components, namely the E- and B-mode components, respectively

$0 \phi=0 \phi^{\mathrm{E}}+\mathrm{i} 0 \phi^{\mathrm{B}}$.

However, the shear induced by gravitational lensing produces an Emode field only since density (scalar) perturbations cannot induce a parity odd B-mode component. In the absence of systematic effects, we have $0 \phi^{\mathrm{E}}=0 \phi$ and $0 \phi^{\mathrm{B}}=0$. The convergence can also be decomposed into a parity even E-mode component and a parity odd B-mode component

$0 \kappa=0 \kappa^{\mathrm{E}}+\mathrm{i} 0 \kappa^{\mathrm{B}}$,

where the B-mode component is again zero in the absence of systematics effects. While the E-mode convergence field is of most interest in the standard cosmological model, the B-mode convergence field is important for testing for residual systematics. Moreover, Bmodes are also useful in studying exotic cosmological models that exhibit parity violation (e.g. Kaufman, Keating \& Johnson 2016). Theoretical models of intrinsic alignments of galaxies can create $B$ modes ( Crittenden et al. 2001, 2002; Hirata \& Seljak 2004), although the measured level is uncertain (Kirk et al. 2015).

The E-mode convergence field represents a scaled version of the integrated mass distribution and thus mapping the intervening matter distribution is often performed by estimating the convergence field. Since the shear is related to the convergence via the lensing potential through equations (18) and (19), convergence maps can be recovered from the observable shear field, which amounts to solving an inverse problem.

\section{MASS-MAPPING ON THE CELESTIAL SPHERE}

In this section, we describe the process of estimating a convergence field from an observed shear field in the spherical setting. Recovering mass-maps by estimating the convergence field involves solving a spherical inverse problem, as discussed above. First, we define the forward problem in spherical harmonic space and explicitly define the spherical generalization of the KS estimator for solving this inverse problem. Secondly, we present an equivalent real space representation of the spherical mass-mapping inverse problem, where 
it can be seen as a deconvolution problem with a spin kernel. Thirdly, we consider the planar approximation of the full spherical setting, recovering the standard planar KS estimator. Finally, we consider iterative refinements to convergence estimators that account for the fact that it is the reduced shear that is observed, rather than the true underlying shear. A variety of such spherical mass-mapping techniques have recently been applied and contrasted on DES year 3 observational catalogues (see e.g. Jeffrey et al. 2021).

\subsection{Harmonic representation}

Using the harmonic representations of the spin raising and lowering operators, it is straightforward to show that the harmonic representations of the convergence and cosmic shear of equations (18) and (19) read, respectively

$0 \hat{\kappa}_{\ell m}=-\frac{1}{2} \ell(\ell+1) 0 \hat{\phi}_{\ell m}$

and

$2 \hat{\gamma}_{\ell m}=\frac{1}{2} \sqrt{\frac{(\ell+2) !}{(\ell-2) !}} 0 \hat{\phi}_{\ell m}$,

where $0 \hat{\phi}_{\ell m}$ and $0 \hat{\kappa}_{\ell m}$ are the scalar spherical harmonic coefficients of the lensing potential and the converge field, respectively, and $2 \hat{\gamma}_{\ell m}$ are the spin-2 spherical harmonic coefficients of the cosmic shear field, i.e. $0 \hat{\phi}_{\ell m}=\left\langle 0 \phi, Y_{\ell m}\right\rangle, 0 \hat{\kappa}_{\ell m}=\left\langle 0 \kappa, Y_{\ell m}\right\rangle$, and $2 \hat{\gamma}_{\ell m}=\left\langle 2 \gamma, 2 Y_{\ell m}\right\rangle$. It follows that the spin- 2 harmonic coefficients of the shear are related to the scalar harmonic coefficients of the convergence by

$2 \hat{\gamma} \ell m_{\ell}=\mathcal{D}_{\ell} 0 \hat{\kappa}_{\ell m}$

where we define the kernel

$\mathcal{D}_{\ell}=\frac{-1}{\ell(\ell+1)} \sqrt{\frac{(\ell+2) !}{(\ell-2) !}}$.

Recovering the convergence field from the observable shear field therefore amounts to solving the inverse problem defined by equation (24). The simplest method to invert this problem is to consider a direct inversion in harmonic space. In the planar setting, such an approach gives rise to the KS estimator (Kaiser \& Squires 1993). An analogous approach in the full-sky setting leads to the spherical generalization of the KS estimator, defined by

${ }_{0} \hat{\kappa}_{\ell m}^{\mathrm{SKS}}=\mathcal{D}_{\ell}^{-1} 2 \hat{\gamma}_{\ell m}^{\mathrm{est}}$,

where $\hat{\gamma}_{\ell m}^{\text {est }}$ denotes the estimate of the shear harmonic coefficients computed from observational data and ${ }_{0} \hat{\kappa}_{\ell m}^{\mathrm{SKS}}$ is the spherical KaiserSquires (SKS) estimator of the harmonic coefficients of the convergence field. A spherical convergence map $0 \kappa^{\mathrm{SKS}}(\omega)$ can then be recovered by an inverse scalar spherical harmonic transform, following equation (8), from which the E- and B-mode components can be determined by considering the real and complex components, following equation (21).

It is well-known that a direct Fourier inversion approach to solving inverse problems, on which the KS estimator is based, is susceptible to noise. On large scales, one typically draws a central limit theory argument for noise Gaussianity, in which case a mutlivariate Gaussian noise model is adopted. In such settings, the KS approach is straightforwardly given by the maximum likelihood estimator, which implicitly assumes a uninformative flat prior. This, combined with the fact that the KS inversion kernel defined by equation (24) has a flat frequency response, indicates that noise present in the observational data set propagates unchecked into the convergence estimate.
Typically one may wish to adopt more informative priors, within a Bayesian setting, to regularize this noise contribution (see e.g. Pichon et al. 2010; Price et al. 2021, where Gaussian and wavelet sparsity priors are adopted respectively). However, for the KS approach, the recovered convergence field is, somewhat naively, smoothed with a Gaussian kernel to mitigate the impact of noise. In this paper, we adopt this post-processing Gaussian smoothing approach and leave more advanced alternatives to future research.

\subsection{Real space representation}

It is insightful to express the forward problem connecting the observable cosmic shear and the convergence field in real space. The differential form of this problem is readily apparent from equations (18) and (19), from which it follows that

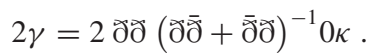

An integral form can also be recovered, where the real space spin-2 shear field is related to the scalar convergence by a type of spherical convolution with a spin-2 kernel $2 \mathcal{K}$

$2 \gamma(\omega)=\int_{\mathbb{S}^{2}} \mathrm{~d} \Omega\left(\omega^{\prime}\right)\left(\mathcal{R}_{\omega^{\prime}} 2 \mathcal{K}\right)(\omega) 0 \kappa\left(\omega^{\prime}\right)$,

where the rotation operator $\mathcal{R}_{\omega^{\prime}}$ is defined in Section 2.2. From comparison with equation (27), it is apparent that the kernel is given by

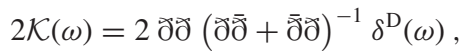

where $\delta^{\mathrm{D}}(\omega)$ is the Dirac delta function on the sphere defined in Section 2.3. Noting the spherical harmonic representation of the Dirac delta function of equation (14) and the harmonic action of the spin raising and lowering operators of equations (4) and (5), it is straightforward to show that the harmonic coefficients of the kernel read

$2 \mathcal{K}_{\ell m}=\frac{-1}{\ell(\ell+1)} \sqrt{\frac{(\ell+2) !}{(\ell-2) !}} \sqrt{\frac{2 \ell+1}{4 \pi}} \delta_{m 0}$.

An explicit expression for the kernel in real space can then be recovered from its harmonic representation, yielding

$2 \mathcal{K}(\omega)=\sum_{\ell} \frac{-1}{\ell(\ell+1)} \frac{2 \ell+1}{4 \pi} P_{\ell}^{2}(\cos \theta)$,

where $P_{\ell}^{2}(\cdot)$ is the associated Legendre function of order two. The equivalence of the harmonic and real space expressions of the forward problem of equations (24) and (28), respectively, can also be seen by the explicit harmonic representation of equation (28), as shown in Appendix A.

\subsection{Planar approximation}

We now consider the planar approximation of the spherical massmapping estimator presented in Section 3.1, recovering the standard planar KS estimator (Kaiser \& Squires 1993). First, we note the planar approximations of the spin raising and lowering operators given by

$\widetilde{\partial} \approx-\left(\partial_{x}+\mathrm{i} \partial_{y}\right)$

and

$\overline{\mathrm{d}} \approx-\left(\partial_{x}-\mathrm{i} \partial_{y}\right)$, 
respectively (see e.g. Bunn et al. 2003). In the planar approximation, the convergence and cosmic shear are then related to the lensing potential by

$0 \kappa=\frac{1}{4}(\check{\partial} \bar{\partial}+\bar{\partial} \check{\partial}) 0 \phi \approx \frac{1}{2}\left(\partial_{x}^{2}+\partial_{y}^{2}\right) 0 \phi$

and

$2 \gamma=\frac{1}{2} ð \partial 0 \phi \approx\left[\frac{1}{2}\left(\partial_{x}^{2}-\mathrm{i} \partial_{y}^{2}\right)+\mathrm{i} \partial_{x} \partial_{y}\right] 0 \phi$,

respectively. It is common to decompose the shear component into its real and imaginary component by

$2 \gamma=\gamma_{1}+\mathrm{i} \gamma_{2}$.

The planar Fourier representations of equations (34) and (35) are then given by

$0 \hat{\kappa}\left(k_{x}, k_{y}\right)=-\frac{1}{2}\left(k_{x}^{2}+k_{y}^{2}\right) 0 \hat{\phi}\left(k_{x}, k_{y}\right)$

and

$\hat{\gamma}_{1}\left(k_{x}, k_{y}\right)=-\frac{1}{2}\left(k_{x}^{2}-k_{y}^{2}\right) 0 \hat{\phi}^{\mathrm{E}}\left(k_{x}, k_{y}\right)+k_{x} k_{y} 0 \hat{\phi}^{\mathrm{B}}\left(k_{x}, k_{y}\right)$,
$\hat{\gamma}_{2}\left(k_{x}, k_{y}\right)=-k_{x} k_{y} 0 \hat{\phi}^{\mathrm{E}}\left(k_{x}, k_{y}\right)-\frac{1}{2}\left(k_{x}^{2}-k_{y}^{2}\right) 0 \hat{\phi}^{\mathrm{B}}\left(k_{x}, k_{y}\right)$,

respectively, where $\hat{\imath}$ denotes the Fourier transform and $k_{x}$ and $k_{y}$ denote the Fourier coordinates, and we make use of the Fourier derivative property $\widehat{\partial_{x} f}=\mathrm{i} k_{x} \hat{f}$. It follows that under the planar approximation, the shear can be related to the convergence in Fourier space by

$2 \hat{\gamma}\left(k_{x}, k_{y}\right)=\mathcal{E}_{k_{x}, k_{y}} 0 \hat{\kappa}\left(k_{x}, k_{y}\right)$,

where

$\mathcal{E}_{k_{x}, k_{y}}=\frac{k_{x}^{2}-k_{y}^{2}+\mathrm{i} 2 k_{x} k_{y}}{k_{x}^{2}+k_{y}^{2}}$.

Analogous to the spherical setting considered in Section 3.1, in the planar setting recovering the convergence field from the shear amounts to solving the inverse problem defined by equation (39). Again, the simplest method to invert this problem is to perform a direct inversion in harmonic space, which gives rise to the standard planar KS estimator (Kaiser \& Squires 1993) of

${ }_{0} \hat{\kappa}^{\mathrm{KS}}\left(k_{x}, k_{y}\right)=\mathcal{E}_{k_{x}, k_{y}}^{-1} 2 \hat{\gamma}^{\text {est }}\left(k_{x}, k_{y}\right)=\mathcal{E}_{k_{x}, k_{y}}^{*}{ }_{2} \hat{\gamma}^{\text {est }}\left(k_{x}, k_{y}\right)$,

where we have taken advantage of the fact that $\mathcal{E}_{k_{x}, k_{y}}^{-1}=\mathcal{E}_{k_{x}, k_{y}}^{*}$ since $\left|\mathcal{E}_{k_{x}, k_{y}}\right|=1$. Recall that ${ }_{2} \hat{\gamma}^{\text {est }}\left(k_{x}, k_{y}\right)$ is the estimate of the planar Fourier coefficients of the shear computed from observational data. Expanding the real and imaginary components, one recovers the familiar KS estimators for the E- and B-mode component of the convergence given by

${ }_{0} \hat{\kappa}^{\mathrm{E}, \mathrm{KS}}\left(k_{x}, k_{y}\right)=\frac{\left(k_{x}^{2}-k_{y}^{2}\right)_{2} \hat{\gamma}_{1}^{\mathrm{est}}\left(k_{x}, k_{y}\right)+2 k_{x} k_{y}{ }_{2} \hat{\gamma}_{2}^{\mathrm{est}}\left(k_{x}, k_{y}\right)}{k_{x}^{2}+k_{y}^{2}}$

and

${ }_{0} \hat{\kappa}^{\mathrm{B}, \mathrm{KS}}\left(k_{x}, k_{y}\right)=\frac{-2 k_{x} k_{y}{ }_{2} \hat{\gamma}_{1}^{\mathrm{est}}\left(k_{x}, k_{y}\right)+\left(k_{x}^{2}-k_{y}^{2}\right)_{2} \hat{\gamma}_{2}^{\text {est }}\left(k_{x}, k_{y}\right)}{k_{x}^{2}+k_{y}^{2}}$,

respectively. A planar convergence map $0 \kappa^{\mathrm{KS}}(\omega)$ can then be recovered by an inverse Fourier transform.

In the above derivation, we have not considered the practicalities of the projection of the fields considered, which are defined natively on the celestial sphere, on to a planar region. In practice, one must choose a specific projection, the choice of which can have a large impact on the quality of the convergence map recovered from the observed shear. We describe a variety of projections in Appendix B and discuss their properties. Care must be taken when projecting a spin-2 field such as the cosmic shear as local rotations must be taken into account, as described in detail in Appendix B.

\subsection{Reduced shear}

In deriving the estimators presented in Sections 3.1 and 3.3, we made the assumption that one could observe the pixelized shear field directly. However, in practice, one can only measure the pixelized reduced shear $_{2} g$, which is related to the true underlying shear by

${ }_{2} g=\frac{2 \gamma}{1-{ }_{0} \kappa}$.

The problem of recovering the convergence field then becomes nonlinear. However, this non-linear problem can be solved iteratively (Seitz \& Schneider 1995; Mediavilla et al. 2016, p.153), as discussed below. These techniques and similar are in common use in the literature (e.g. Jullo et al. 2014; Lanusse et al. 2016; Price et al. 2021)

The first step is to denoise the map of reduced shear. In this work, we use a Gaussian smoothing. We make an initial estimate of the shear by assuming it is simply the measured reduced shear. Then an initial estimate of the pixelized convergence field is made. The first step of the iterative algorithm is thus

$$
\begin{aligned}
& { }_{2} \gamma^{(0)}={ }_{2} g, \\
& { }_{0} \kappa^{(0)}=M\left[{ }_{2} \gamma^{(0)}\right],
\end{aligned}
$$

where $M$ denotes the mass-mapping estimator used to recover the convergence from the shear (in this article, we consider either the spherical or planar KS estimators described in Sections 3.1 and 3.3, respectively) and the superscript denotes iteration number. We then use our estimate of the convergence to update the estimate of the shear and repeat. The $(i+1)$-th iteration is thus

$$
\begin{aligned}
& { }_{2} \gamma^{(i+1)}={ }_{2} g\left(1-{ }_{0} \kappa^{(i)}\right), \\
& { }_{0} \kappa^{(i+1)}=M\left[{ }_{2} \gamma^{(i+1)}\right] .
\end{aligned}
$$

Iterations are continued until the absolute difference of the convergence between iterations is below some threshold value. In this work, we choose

$$
\max _{j}\left|{ }_{0} \kappa_{j}^{(i)}-{ }_{0} \kappa_{j}^{(i-1)}\right|<10^{-10},
$$

where $j$ runs over all pixels. Typically, for a convergence field including ellipticity/shot noise, 4-5 iterations are required before converging.

\subsection{Implementation}

We have written the PYTHON package massmappy ${ }^{8}$ to implement the algorithms presented. The package can perform standard massmapping on the plane, with the option to perform iterations to account for reduced shear. We also implement the SKS estimator described above so that mass-mapping can be performed on the celestial sphere. We support the use of two spherical pixelizations schemes. First, we support the use of HEALPix ${ }^{9}$ (Górski et al. 2005), an equal area pixelization with an accompanying software package that can

\footnotetext{
${ }^{8}$ http://www.massmappy.org

${ }^{9}$ http://healpix.jpl.nasa.gov
} 
perform fast spherical harmonic transforms. We also support the use of the standard equiangular sampling scheme implemented in SSHT $^{10}$ (McEwen \& Wiaux 2011). This sampling scheme supports fast spherical harmonic transforms that are theoretically exact and achieve close to floating point precision in practice. The most recent release of SSHT includes fast routines to compute the projections of the sphere on to the plane considered in this work.

\section{EVALUATION ON SIMULATIONS}

In this section, we evaluate the mass-mapping algorithms presented in Section 3 on simulations. We study the error introduced by the planar approximation, for a variety of projections and for varying survey coverage area, when compared to the spherical setting. We also assess the ability of the iterative algorithm described in Section 3.4 to deal with the reduced shear that is observed, rather than the underlying true shear.

\subsection{Comparison of planar and spherical mass-mapping}

We study the impact of the flat-sky planar approximation in massmapping, compared to the spherical setting, and determine the typical errors induced for the sky coverages of upcoming surveys. We do this as an idealized situation to focus the study on the effect of projecting the sphere on to the plane. To do so, we need to understand how best one can estimate mass-maps on the plane for large coverage areas.

When creating convergence maps on the plane (i.e. mass-maps), the exact projection used to map the celestial sphere to the plane can have a large impact on the quality of the reconstructed convergence map. In Appendix B, we describe a variety of spherical projections that can be considered, which we evaluate on simulations here. One important aspect when projecting a non-zero spin field, e.g. shear (or galaxy ellipticities), is to ensure that the correct local rotations are performed, as described in Appendix B2. This is typically neglected in existing mass-mapping works.

We now describe the simulations that we use to assess the effect each projection has on the quality of the reconstruction of convergence maps. We simulate Gaussian convergence maps using a convergence power spectrum generated by the software package COSMOSIS $^{11}$ (Zuntz et al. 2015). The power spectrum was generated with a standard $\Lambda \mathrm{CDM}$ cosmology with galaxies in high redshift bin $z \gtrsim 1$. We simulate the map up to a harmonic band-limit of $\ell_{\max }=512$ using the sampling of the sphere of SSHT (McEwen \& Wiaux 2011). We consider this spherical sampling scheme for these numerical experiments since the resulting spherical harmonic transforms are theoretically exact and the implementations in SSHT achieve accuracy close to machine precision (which is not the case for HEALPix; see Leistedt et al. 2013 for concise accuracy benchmarks). Any errors will therefore be due to projection effects rather than inaccuracies in harmonic transforms. We smooth the simulated convergence maps with the Gaussian kernel $G_{\ell}=e^{-\ell^{2} \sigma^{2}}$, with $\sigma=\pi / 256$, to mitigate pixelization issues. The shear field is simulated by transforming the scalar convergence field to harmonic space and then applying equation (24), before transforming back to real space to recover a spin- 2 shear field on the celestial sphere. In these simulations, we aim to understand the effect of the projections so we do not consider the effects of reduced shear or noise.

\footnotetext{
${ }^{10}$ http://www.spinsht.org

${ }^{11}$ https://bitbucket.org/joezuntz/cosmosis/wiki/Home
}

To evaluate the accuracy of planar mass-mapping, we first project the simulated shear field from the celestial sphere to the plane, using a particular projection. We estimate the convergence field from the planar shear field using the planar KS estimator of equation (41). We then compare this recovered planar convergence to a planar projection of the convergence simulated initially on the celestial sphere. A number of different projections are considered, as defined in Appendix B. In general, we consider two classes of spherical projection: namely, equatorial and polar projections.

In Fig. 2, we show example planar reconstructions and errors for a variety of equatorial projections. These projections are highly accurate on the equator, with distortion due to the projection typically increasing with distance from the equator. We consider, first, a simple cylindrical projection, where the $(\theta, \varphi)$ angles are taken to be Cartesian coordinates $(x, y)$. We also consider the Mercator projection, which is often used for geographical maps. The Mercator projection is a conformal projection, in that it preserves local angles. The poles in this projection would be at infinity, so we limit the projection to $7 \pi / 16$ radians above and below the equator. Finally, Fig. 2 shows results using the sinusoidal projection, a simple equal area projection used by the DES collaboration for the convergence map generated from DES SV data (Vikram et al. 2015).

In Fig. 3, we show example planar reconstructions and errors for a variety of polar projections. These projections are highly accurate around the pole defining the centre of the projection, with distortion increasing as one moves away from this point. For these projections, we project one hemisphere around a pole defined by the $x$-axis only; hence, two projections (one for each hemisphere) are required to cover the entire sphere. ${ }^{12}$ We consider the orthographic projection, which is a simple vertical projection, the stereographic projection, which is another conformal projection, and finally the Gnomonic projection, which has the special property that the local rotations required for the projection of spin fields are zero (if no coordinate rotation is performed). The edge of the hemisphere for the Gnomonic projection lies at infinity so we only project the sphere on to the square where the distance from the centre of the square and its edge represents an angle of $\pi / 4$ radians.

For all projections, we show in Figs 2 and 3 the projected shear, the recovered $E$-mode convergence, and the error in the $E$ - and $B$ mode convergence. As expected, the convergence reconstruction is best where the planar approximation is most accurate and worse as one moves away from this region. We can also see by eye that the conformal projections (the Mercator and stereographic projections) perform the best. This is due to fact that local angles are preserved by the projection. What is also clear is that for many of the projections, the $B$-mode convergence error can be large in certain regions even in the absence of noise or systematic errors.

We can use these simulations to examine the error in the reconstructed convergence field as a function of angular size. In Fig. 4, we show how the accuracy of the recovered convergence field changes with patch size. We consider a similar simulation setup as the low resolution experiments described above but now simulate the convergence field up to a band limit $\ell_{\max }=4000$, using the same power spectrum and smoothing kernel as before. We set a higher band-limit to eliminate all pixelization effects (a lower band-

\footnotetext{
${ }^{12}$ For the stereographic projection, a single projection can be applied to map the sphere to the plane. However, the opposite pole is mapped to the point at infinity. Moreover, the size of the planar regions grows considerably as the full coverage of the celestial sphere is approached. Consequently, for practical purposes, the two hemispheres are projected separately.
} 


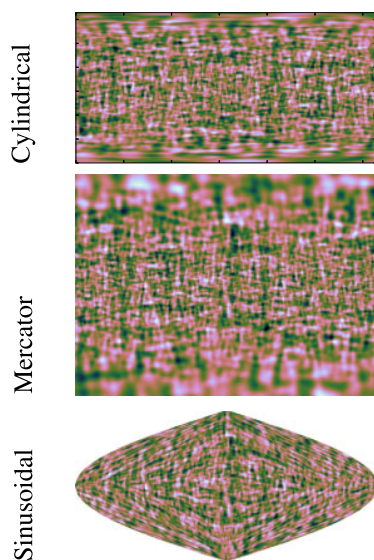

(a) $\gamma_{1}$
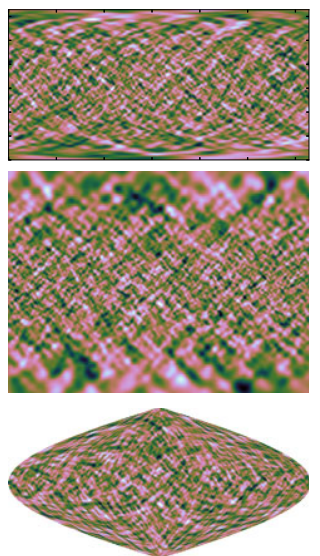

(b) $\gamma_{2}$
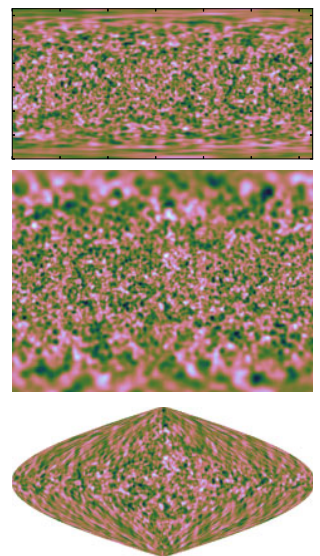

(c) $\kappa^{\mathrm{E}, \mathrm{KS}}$
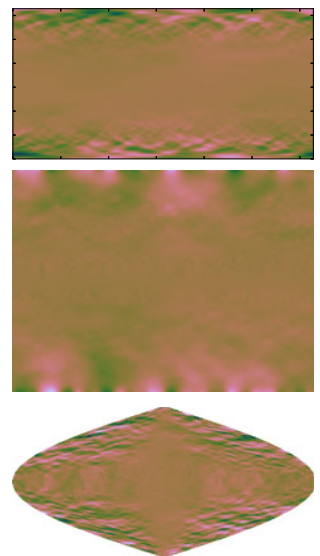

(d) $\kappa^{\mathrm{E}, \mathrm{KS}}$ error
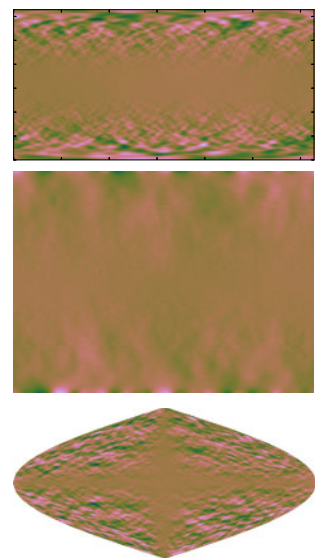

(c) $\kappa^{\mathrm{B}, \mathrm{KS}}$ error

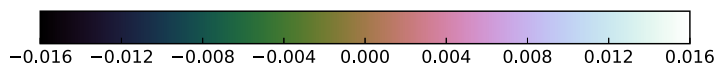

Figure 2. Simulated reconstructions of the convergence field (mass-maps) on large regions of the celestial sphere when using equatorial projections, in order to assess the impact of different planar projections. The shear field is shown in the first and second columns (the first showing $\gamma_{1}$ and the second showing $\gamma_{2}$ ). The third column shows the reconstructed convergence field ( $E$-mode), while the forth and fifth columns show the error on the $E$-mode and $B$-mode convergence, respectively. Each row shows a different projection: the first row shows the simple cylindrical projection; the second shows the Mercator projection; and the final row shows the sinusoidal projection. The entire sphere is projected on to the plane, except for the Mercator projection where only $7 \pi / 16$ radians above and below the equator are considered (as explained in the main text).

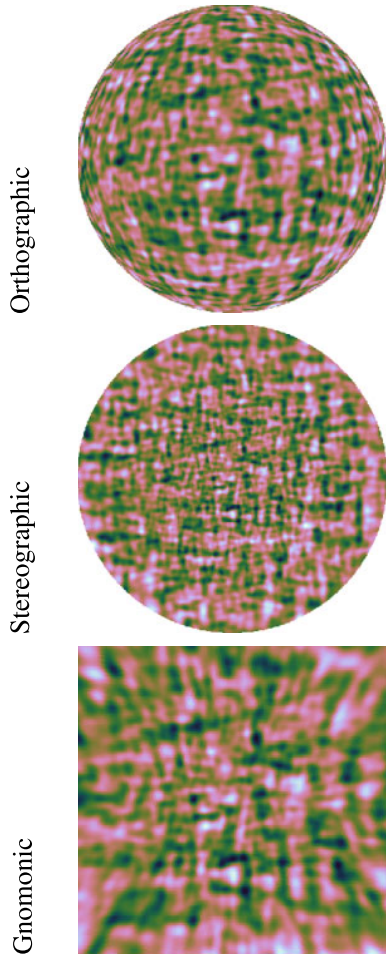

(a) $\gamma_{1}$
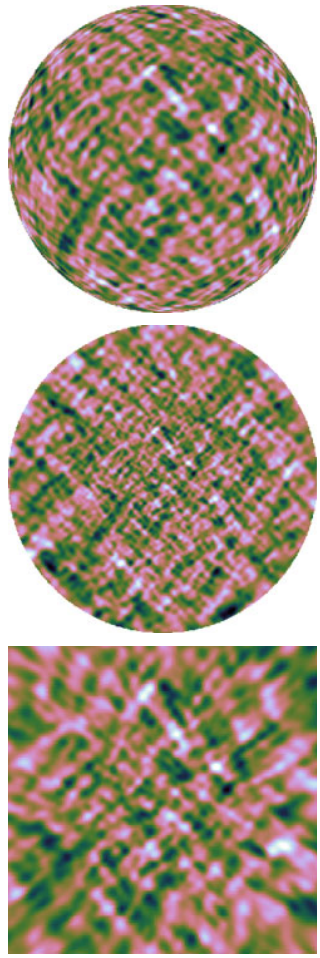

(b) $\gamma_{2}$
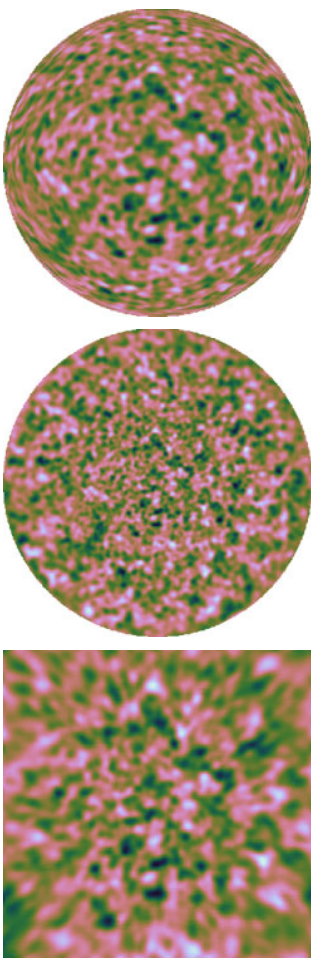

(c) $\kappa^{\mathrm{E}, \mathrm{KS}}$
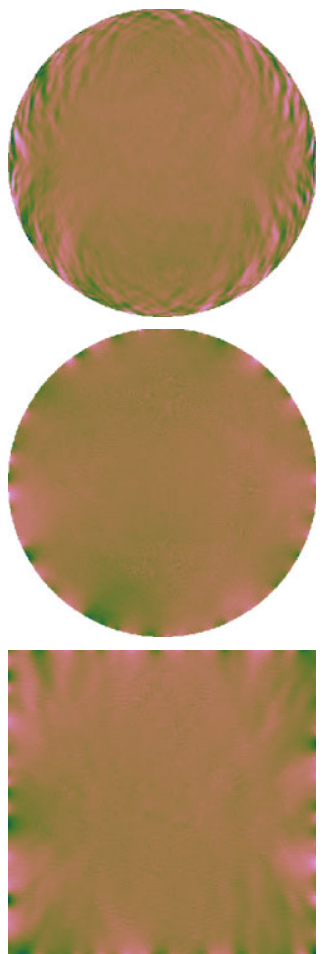

(d) $\kappa^{\mathrm{E}, \mathrm{KS}}$ error
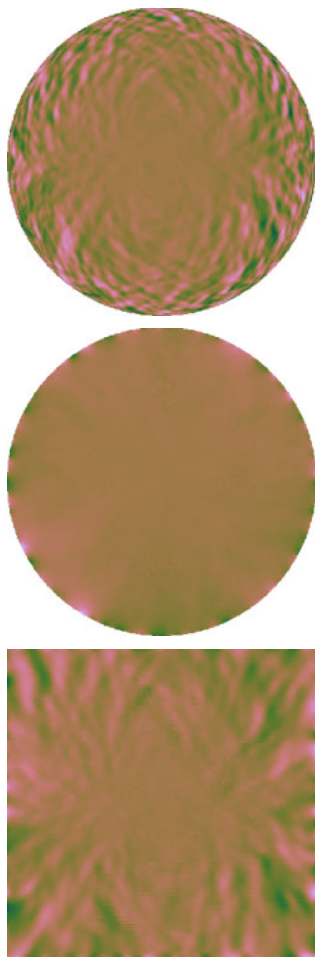

(c) $\kappa^{\mathrm{B}, \mathrm{KS}}$ error

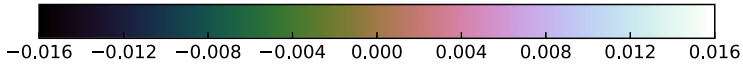

Figure 3. Same as Fig. 2 for the polar projections. The first row shows the orthographic projection, the middle row shows the stereographic projection, and the third row shows the Gnomonic projection. For these projections, we only project one hemisphere on to the sphere, with the pole defined by the $x$-axis. The entire hemisphere is shown except for the Gnomonic projection where we project the sphere on to a square where distance from the centre of the square and the edge represents an angle of $\pi / 4$ radians (as explained in the main text). Of course, planar approaches are typically restricted to a field of view of $\sim 20^{\circ}$, these figures simply illustrate why this consensus is adhered to. 


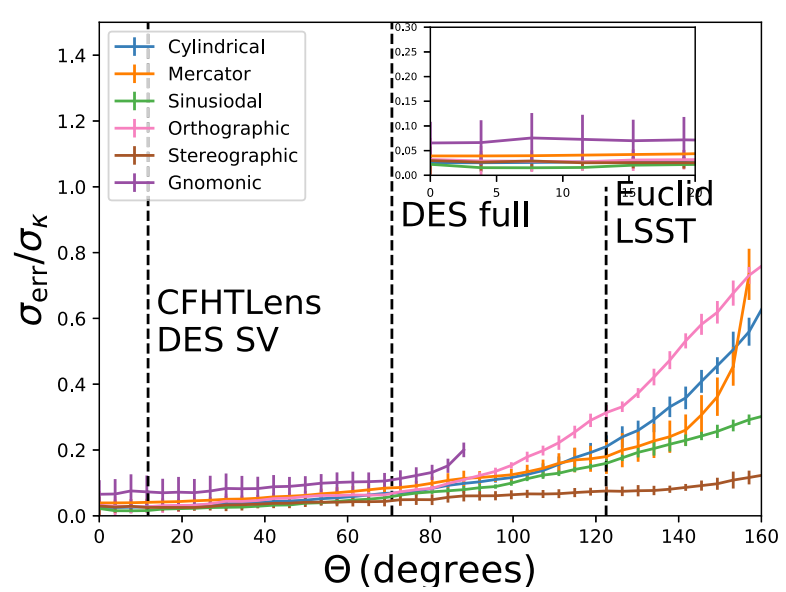

(a) $\kappa^{\mathrm{KS}} E$-mode error

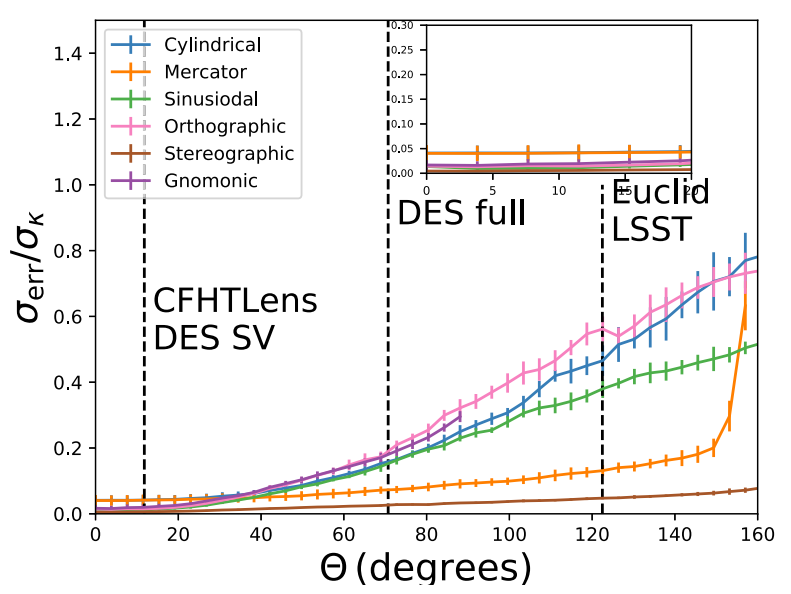

(b) $\kappa^{\mathrm{KS}} B$-mode error

Figure 4. Relative rms error of recovered convergence fields (mass-maps) when using various planar projections in the standard planar KS estimator, as a function of angular distance from the centre of the projection. Note that all planar projections were significantly zero-padded (to four times their original dimension) to minimize any contribution to this error from mode mixing at boundaries. Further, we note that residual boundary effects inevitably exist, thus we clip the figure $20^{\circ}$ from any boundary, thus restricting the figure to $\Theta$ domains over which it is expected the primary error contribution comes from projection effects. rms errors are averaged over 10 realizations. Approximate opening angles for the coverages of existing and upcoming surveys are overlaid. For future surveys, such as Euclid and LSST, projection errors can be of order tens of percent, exceeding 50 percent in some cases. The conformal projections (i.e. the Mercator and stereographic projections), which preserve local angles, are typically superior to the other projections. In any case, these errors can be avoided entirely by recovering convergence fields directly on the celestial sphere.

limit was sufficient for the previous numerical experiments which were used for visualization purposes only). We oversample on the plane too, again to eliminate all pixelization effects. For the polar projections, we use a square map of $2000 \times 2000$ pixels, capturing the same hemisphere as before. For the equatorial projections, we use maps of size $\left(2 \ell_{\max }-1\right) \times \ell_{\max }$ pixels for the cylindrical projection, $\left(2 \ell_{\max }-1\right) \times 5901$ pixels for the Mercator projection, and $\left(2 \ell_{\max }-1\right) \times \ell_{\max }$ pixels for the sinusoidal projection. The number of pixels is different of the Mercator projection as it stretches the $\theta$ direction in projection. The equatorial projections, as before, have the entire sphere projected on to the plane except for the Mercator projection where we project to $7 \pi / 16$ radians above and below the equator only as the poles are at infinity in this projection. The exact planar sampling resolutions are not important as we are intentionally oversampling to eliminate pixelization effects.

In a similar way to the other simulations we simulate the convergence and shear on the sphere, project the shear on the plane, and recover the convergence on the plane to compare this to the projected simulated convergence. We then calculate the root-mean-square (rms) error of $\frac{1}{N} \sum_{i}^{N}\left(\kappa^{\mathrm{KS}}-\kappa^{\text {input }}\right)^{2}$ at different angular distances from the most accurate region of each projection, where $N$ is the number of pixels in the region and $\kappa^{\text {input }}$ is the input convergence. The exact angular distances considered for each projection are defined in Appendix B. We calculate the error in annuli of constant angular distances away from the centre, defined by the angular metric. The error in the recovered convergence will be a result of not only the projection distortion but also a sub-dominant contribution from the leakage due to the boundary created by the projection. The leakage due to boundary effects will be minimal for small and intermediate scales but will become more significant for the largest scales considered - i.e. as the annuli approach the boundary region. Both projection and boundary effects are intrinsic to the projection when using KS inversion and are therefore included here. To minimize the contribution of such boundary effects, we zeropad planar projections to four times their original dimensions, and restrict any analysis to annuli separated by at least $20^{\circ}$ from any boundaries.

Fig. 4 shows the rms error, averaged over 10 realizations, at different angular distances for the various projections considered. We normalize the rms error with the rms of the fluctuations in that region to give a relative error. Relative error for both the $E$ and $B$-modes fields are shown. Approximate opening angles for the coverages of existing and upcoming surveys are overlaid on Fig. 4. For future surveys, such as Euclid and LSST, projection errors can be of the order of tens of percent, exceeding 50 percent in some cases. The conformal projections (i.e. the Mercator and stereographic projections), which preserve local angles, are typically superior to the other projections. In any case, these errors can be avoided entirely by recovering convergence fields directly on the celestial sphere.

\section{APPLICATION TO DES SV DATA}

In this section, we apply the mass-mapping techniques presented in Section 3 to the DES SV data, which are publicly available. ${ }^{13}$ We use the galaxy shapes estimated by the IM3SHAPE method that lie in the range $60^{\circ}<\mathrm{RA}<95^{\circ}$ and $-70^{\circ}<$ Dec. $<-40^{\circ}$, where RA and Dec. are the right ascension and declination in degrees. We apply the sva1_flag $=0$ selection to the DES SV catalogue in order to select galaxies that have a shape that is measured and calibrated ready to be used for weak lensing studies. These cuts leave 793743 galaxies, with a density of 1.4 galaxies per square arcmin. We pixelize the data by binning into pixels in various settings. We always pixelize the galaxy in the space that the convergence map is generated; for example, when a map is made on the sphere the galaxies are pixelated on the sphere directly. In all cases, we apply the recommended weights and corrections to account for multiplicative and additive biases, as described by Becker et al. (2016).

\footnotetext{
${ }^{13}$ https://des.ncsa.illinois.edu/releases/sva1/doc/shear
} 


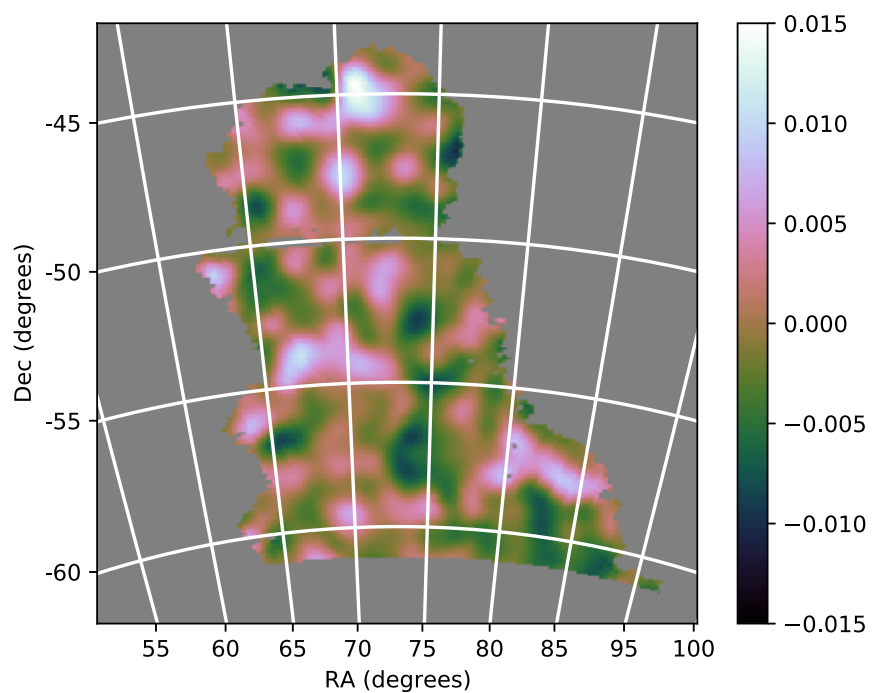

(a) $K^{\mathrm{SKS}} E$-mode (SSHT)

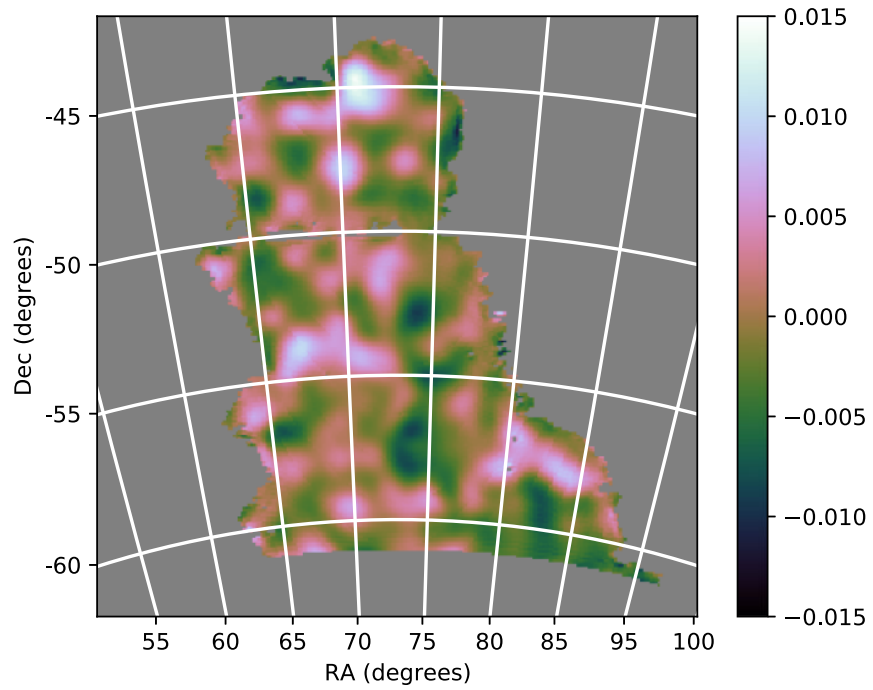

(c) $\kappa^{\mathrm{SKS}} E$-mode (HEALPix)

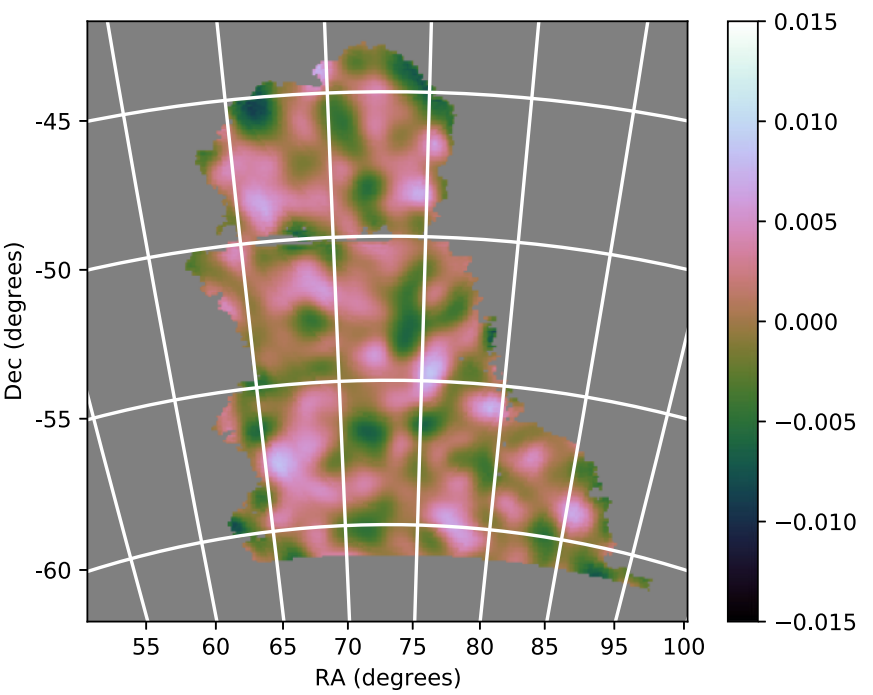

(b) $\kappa^{\mathrm{SKS}} B$-mode (SSHT)

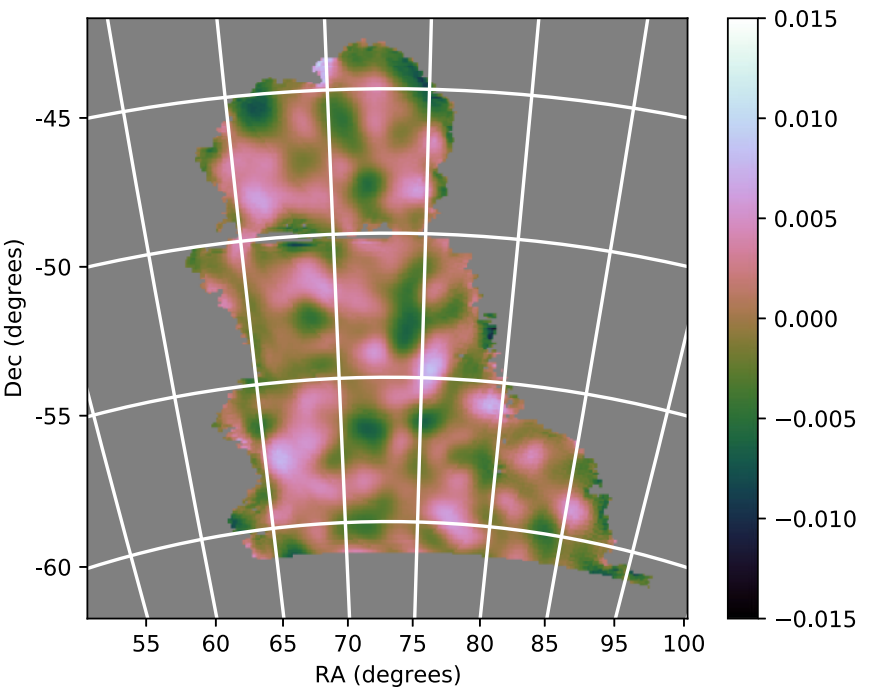

(d) $\kappa^{\text {SKS }} B$-mode (HEALPix)

Figure 5. Spherical convergence maps recovered by the SKS estimator applied to spherical maps of the reduced shear created using galaxies from DES SV data. The top two plots show stereographic projections of the convergence map recovered on the celestial sphere using the SSHT sampling, while the bottom two plots show Stereographic projections of the convergence maps recovered on the celestial sphere using HEALPix sampling. The left-hand column shows the recovered $E$-mode convergence, while the right-hand column shows the recovered $B$-mode convergence. To generate these maps from the DES observation catalogue, we first grid on to a HEALPix sampling scheme, then convert this to a SSHT sampling scheme through harmonic space, thus, both reconstructions are working with the same information which mitigates any discrepancies due to the initial catalogue projection.

We create two spherical maps of the reduced shear using the SSHT and HEALPix sampling schemes, considering resolutions to best match the $\delta \theta=5$ arcmin pixels considered by Vikram et al. (2015), which corresponds to setting an appropriate bandlimit $\ell_{\max }$ for the SSHT sampling scheme and an appropriate $N_{\text {side }}$ resolution parameter for HEALPix. Explicitly, for SSHT, we find $\ell_{\max }=$ $\pi / \delta \theta=2160$. For HEALPix, we set $N_{\text {side }}$ such that the area of a pixel is as close as possible to that of a 5 -arcmin pixel, i.e. $A=4 \pi / 12 N_{\text {side }}^{2} \approx(\delta \theta)^{2}$, yielding $N_{\text {side }}=512$ (with the restriction that $N_{\text {side }}$ is a power of two). The resulting SSHT map has pixels of size 5 arcmin at the equator, while the resulting HEALPix map has pixels of size 7 arcmin. For the HEALPix sampled data, we use a maximum multipole $\ell_{\max }=4 N_{\text {side }}$. The exact choice of $\ell_{\max }$ is not critical as smoothing removes the power on small scales. We smooth the reduced shear before reconstructing the mass-map with a Gaussian Kernel $G_{\ell}=e^{-\ell^{2} \sigma^{2}}$, with $\sigma$ such that the half width at half maxima is 20 arcmin, to best match that of Vikram et al. (2015).

It is academic to note that interpolation errors are effectively unavoidable when mapping observations continuous in position on to a finite grid. Furthermore, gridding on to different sampling schemes inherently introduces different interpolation error. One may wonder, quite reasonably, which sampling (or corrective measure) minimizes this interpolation error, however, this is beyond the scope of this paper. To normalize for this effect within this analysis, we first grid on to HEALP ix map which we then convert into a SSHT sampled map with the aforementioned dimensions. In this way both maps begin with the same information contaminated with the same interpolation error. 


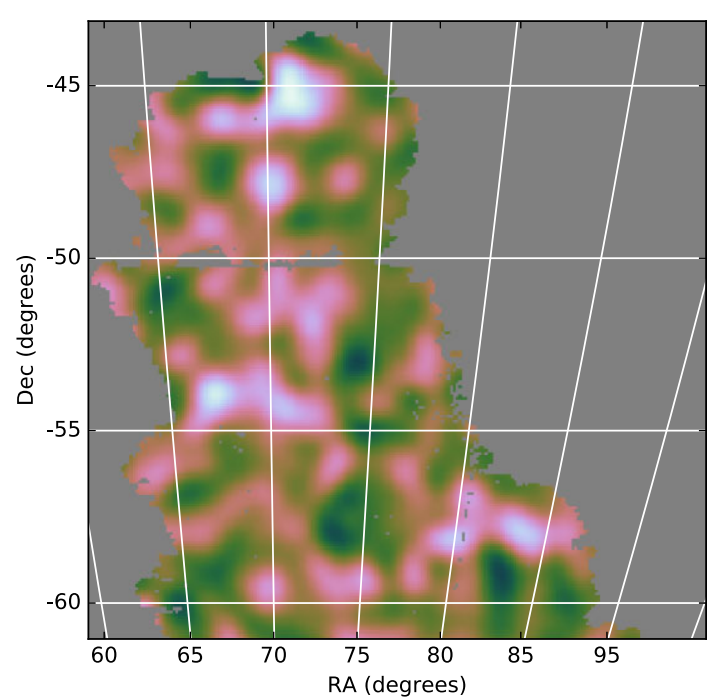

(a) $\kappa^{\mathrm{KS}} E$-mode for sinusoidal projection

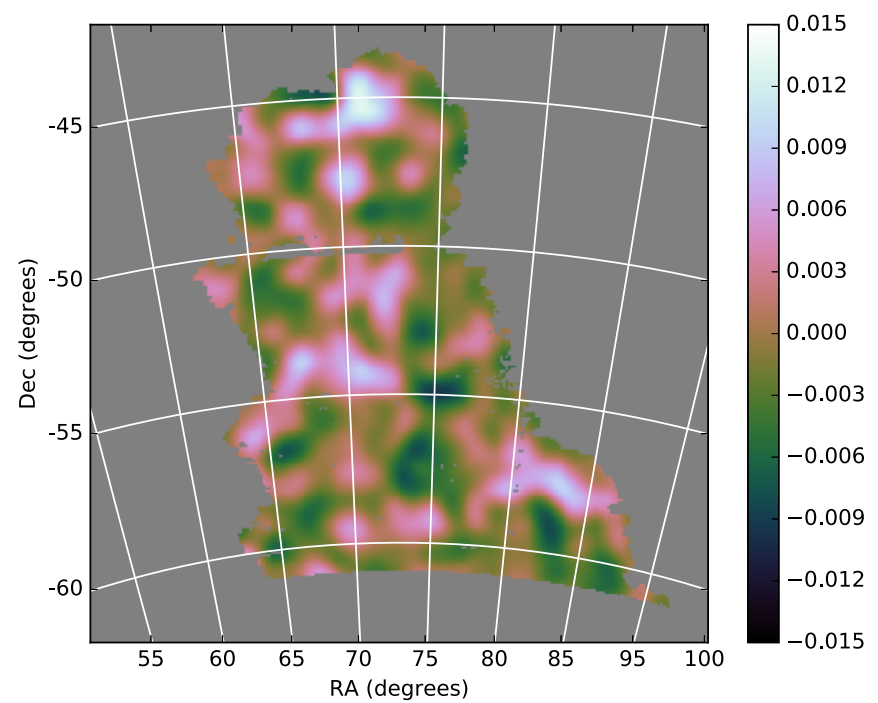

(c) ${ }^{\mathrm{KS}} E$-mode for stereographic projection

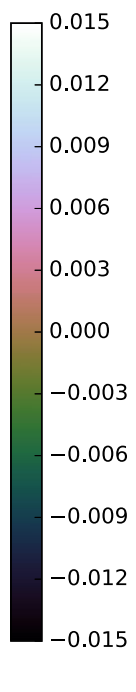

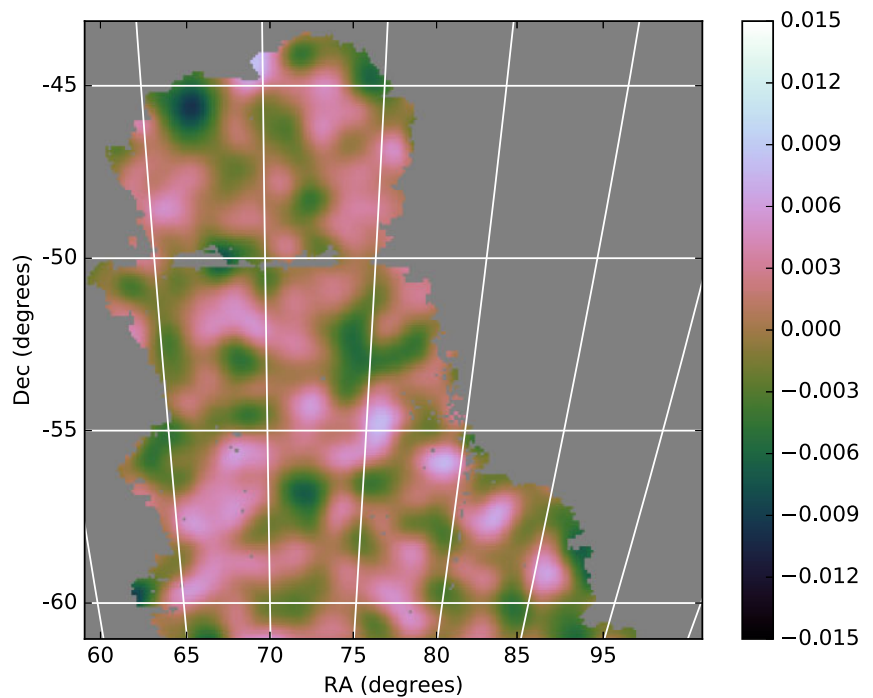

(b) $\kappa^{\mathrm{KS}} B$-mode for sinusoidal projection

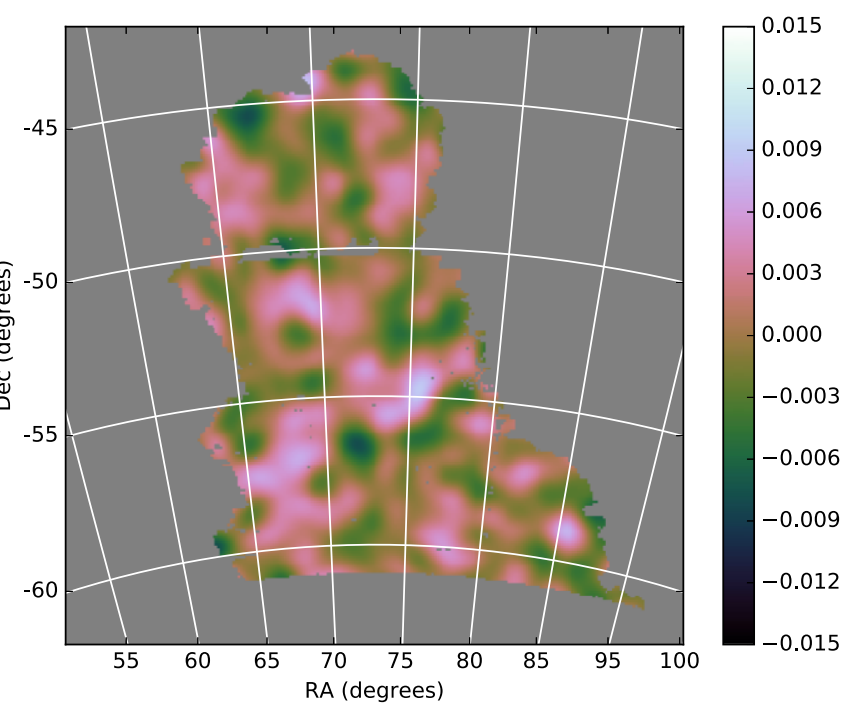

(d) $\kappa^{\mathrm{KS}} B$-mode for stereographic projection

Figure 6. Planar convergence maps recovered by the planar KS estimator applied to planar maps of the reduced shear created using galaxies from the DES SV data. The top row of plots show the results where the sinusoidal projection is used, while the bottom row shows the results when the stereographic projection is used. These projections were chosen since the the sinusoidal projection is used by the DES collaboration (Vikram et al. 2015), while the stereographic projection was shown in Fig. 4 to minimize rms error. The left-hand column shows the recovered $E$-mode convergence, while the right-hand column shows the recovered $B$-mode convergence.

Further, one should note that the noise properties of interpolated spherical maps depend fundamentally on the sampling scheme adopted. When one considers HEALPix equal area sampling, each pixel contains roughly the same number of observations, whereas for SSHT equiangular sampling pixels have significant variation in the number of observations (due to variability in pixel size). As such, the assumption of noise Gaussianity is more easily justified for HEALPix maps.

Fig. 5 shows the $E$ - and $B$-mode convergence maps recovered from the DES SV data using the spherical Kaiser-Squires (SKS) estimators. We apply the iterative algorithm described in Section 3.4 to estimate the underlying shear from the observed reduced shear. The recovered convergence maps show near perfect agreement with each other and reasonable agreement with the maps recovered by the DES collaboration for a similar choice of galaxies (Vikram et al. 2015, fig. 2). It should be noted that the galaxies used here are not the exact same galaxies used in estimating the convergence maps recovered by Vikram et al. (2015) due to small differences between the private and public DES catalogues (C. Chang \& J. Zuntz, private communication). Therefore, exact equivalence is not excepted, however, through private communication, C. Chang has provided convergence maps recovered by the DES map making pipeline when using the public catalogue and in this case, there is good agreement between the two convergence maps.

For comparison purposes, in Fig. 6, we show the results when we bin galaxies on to two planar maps. The top row shows the results when using a sinusoidal projection, as also used by the DES collaboration (Vikram et al. 2015). We rotate the projection such 


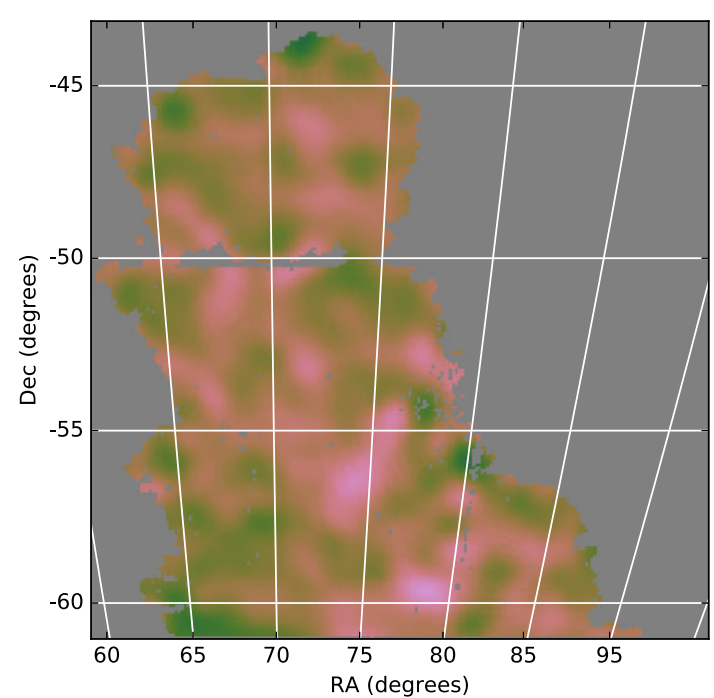

(a) $\kappa^{\mathrm{KS}}-\kappa^{\mathrm{SKS}} E$-mode for sinusoidal projection

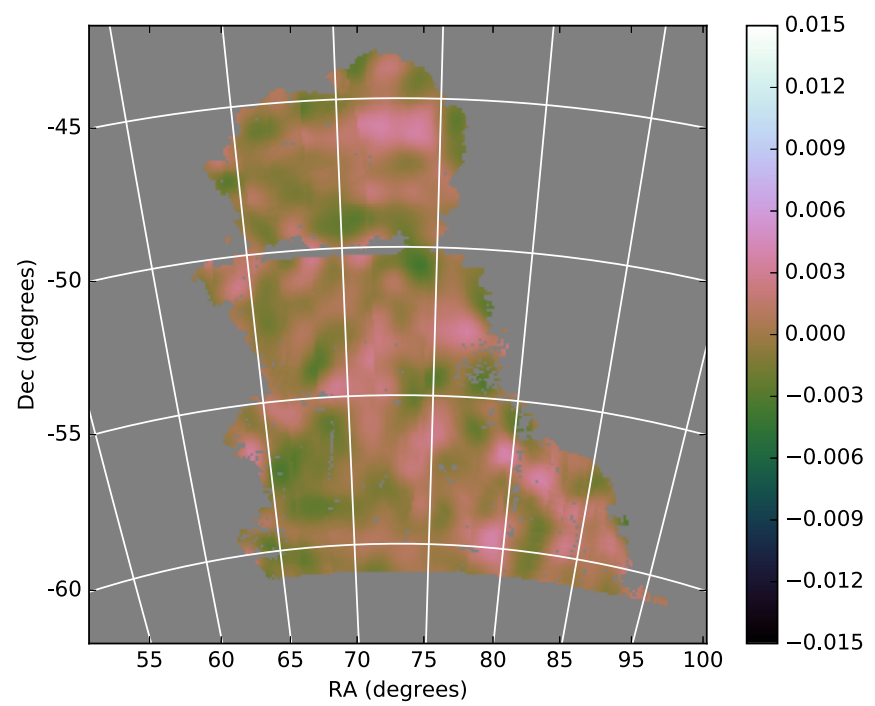

(c) $\kappa^{\mathrm{KS}}-\kappa^{\mathrm{SKS}} E$-mode for stereographic projection

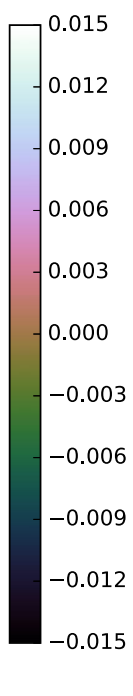

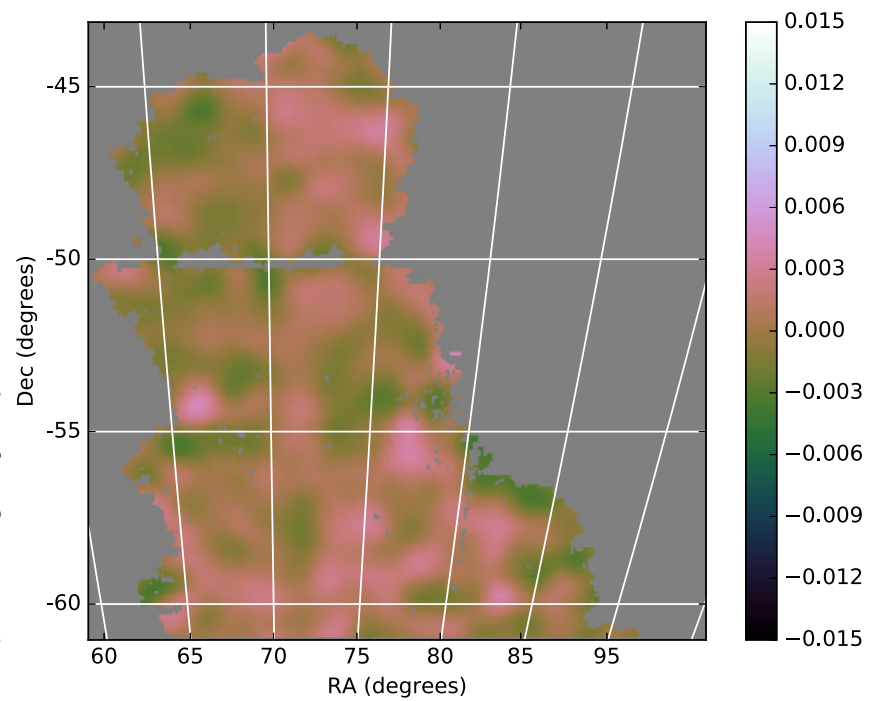

(b) $\kappa^{\mathrm{KS}}-\kappa^{\mathrm{SKS}} B$-mode for sinusoidal projection

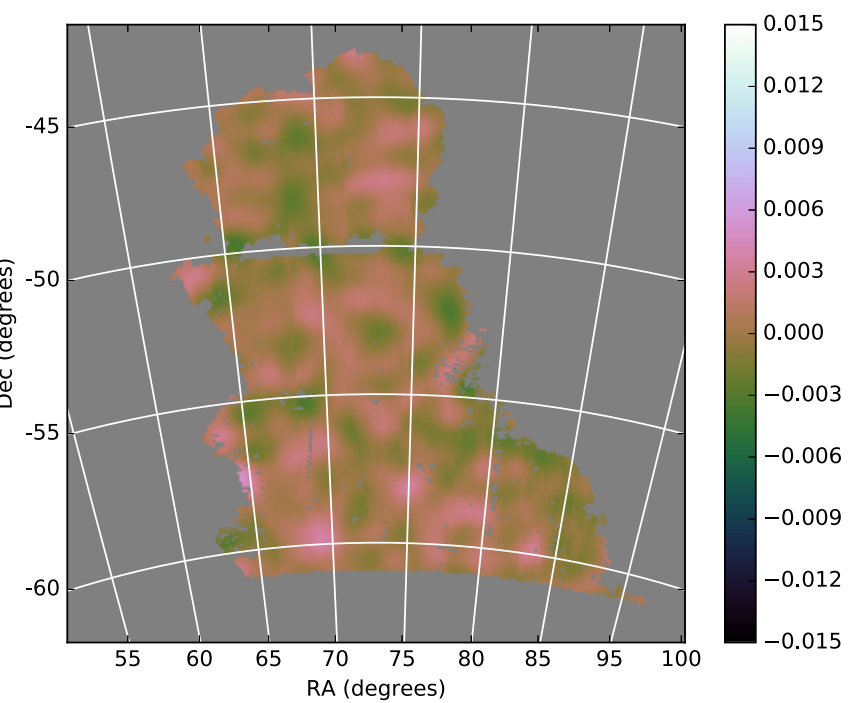

(d) $\kappa^{\mathrm{KS}}-\kappa^{\mathrm{SKS}} B$-mode for stereographic projection

Figure 7. Similar to Fig. 6, where here we plot the difference between the convergence recovered on the plane by the planar KS estimator and the convergence recovered on the sphere by the SKS estimator. The purpose of this figure is to compare the planar and spherical results. For the spherical case, we consider the SSHT sampling only, i.e. differences are relative to Figs 5(a) and (b).

that the central line of the projection corresponds to RA $=70^{\circ}$, as also done by Vikram et al. (2015). No other rotation is applied to fully centre the region of interest. In the second row, we show results using a stereographic projection that has been rotated by the Euler angles $\alpha=159^{\circ}, \beta=-37^{\circ}$, and $\gamma=90^{\circ}$, to fully centre the area of interest to the South Pole about which the projection is then performed. We choose to also show results using the stereographic projection as the results from Fig. 4 suggest that this is the best projection to use. In both cases, we use 5-arcmin pixels and apply a 20-arcmin smoothing as they do in Vikram et al. (2015). We apply the required local rotations as described in Appendix B (in Appendix B, we also examine the effect of not applying such rotations). For these planar results, we also use the reduced shear algorithm described in Section 3.4. Fig. 7 shows the difference between the convergence recovered on the plane for these projections and the projected convergence recovered on the sphere using the SSHT sampling shown in Fig. 5. As is common with the KS estimator, both the planar and the spherical mass-maps suffer from leakage between the $E$ - and $B$-mode due to both the effects of the boundary and, perhaps primarily, the significant complex noise contribution.

\section{CONCLUSIONS}

We have described how one can recover convergence fields, or mass-maps, directly on the celestial sphere, adopting the spherical equivalent of KS inversion. We demonstrate that the spherical formulation reduces to the usual flat-sky KS approach in the planar approximation. We study the accuracy of the planar approximation for mass-mapping and address the important question of whether one needs to recover the convergence field on the sphere for forthcoming surveys or whether recovery on the plane would be sufficient. The comparison between the planar and spherical settings depends largely on the projection used. In Appendix B, we describe a number of projections that are used in this work and show 
how to account for the local rotations required when projecting spin fields, such as shear, on to the plane. In Fig. 4, the relative error introduced by the planar approximation, for a variety of projections, is presented. Conformal projections, for which local angles are conserved, are found to be the most effective. Nevertheless, errors in the planar setting are typically tens of percent and can exceed 50 percent in some cases. These projection errors can be entirely eliminated by recovering mass-maps directly on the celestial sphere by the SKS technique presented in this article. This analysis of projection errors has broader implications for the analysis of signals over the sphere, e.g. cosmic microwave background analysis etc.

We apply the SKS estimator to the publicly available DES SV data. We present maps of the convergence field recovered on the celestial sphere using both the SSHT and HEALPix sampling schemes (see Fig. 5), accounting for the fact that one measures reduced shear, rather than the true underlying shear, by applying the iterative algorithm discussed above. We compare the results to those recovered on the plane, using the sinusoidal projection adopted by the DES collaboration and also the stereographic projection since it was found to be most effective projection for mass-mapping, particularly for large scales (see Fig. 4). In this setting, we demonstrate reasonable agreement between the spherical and planar reconstructions. While the coverage area of DES SV data is not sufficiently large for the planar approximation to induce significant errors (see Fig. 4), recovering spherical mass-maps for DES SV data is nevertheless a useful demonstration of the SKS estimator on real observational data.

In this article, we consider the most naive estimator of the convergence field on the celestial sphere, namely a direct spherical harmonic inversion of the equations relating the observed shear field to the underlying convergence field, i.e. the generalization of the KS estimator from the plane to the sphere. In practice, the shear field is not observed over the entire celestial sphere, which induces leakage in the recovered convergence field for the simple harmonic estimator considered. In future work, we will apply the pure mode wavelet estimators developed by Leistedt et al. (2017) to remove leakage when recovering spherical mass-maps. In addition, in future work, we also intend to develop methods to better mitigate the impact of noise and to estimate the statistical uncertainties associated with recovered mass-maps (see e.g. Price et al. 2020). In all of these extensions, however, it is clear that for future surveys like Euclid and LSST, it will be essential to recover mass-maps on the celestial sphere, to avoid the significant errors than are otherwise induced by planar approximations.

\section{ACKNOWLEDGEMENTS}

We thank the DES team for making their shear data public, and we thank C. Chang and J. Zuntz for assistance in the manipulation of these catalogues. We thank C. Chang for the private communications including providing us with convergence maps created using the publicly available shear catalogues and DES map making pipeline. We thank P. Paykari and Z. Vallis for useful discussions. This work was supported by the Science and Technology Facilities Council (STFC) through a Euclid Science Support Grant and an LSST: UK Phase A grant, the Engineering and Physical Sciences Research Council (EPSRC; grant number EP/M011852/1), and the Leverhume Trust. TDK acknowledges support of a Royal Society University Research Fellowship. It should be noted that this paper was first submitted in 2017, however, due to the lead author leaving academia, the review process was not complete until 2021. During this period, analysis techniques for weak lensing signals over the celestial sphere have evolved substantially, in many regards motivated by the results from this work (see e.g. Chang et al. 2018; Jeffrey et al. 2021; Price et al. 2021)

\section{DATA AVAILABILITY}

All data, both observational and simulated, utilized throughout this paper is publicly available and can be found alongside the opensource code-base mas smappy ${ }^{14}$ developed during this work.

\section{REFERENCES}

Aghanim N., Kunz M., Castro P. G., Forni O., 2003, A\&A, 406, 797

Alsing J., Heavens A., Jaffe A. H., Kiessling A., Wandelt B., Hoffmann T., 2016, MNRAS, 455, 4452

Bacon D. J., Taylor A. N., 2003, MNRAS, 344, 1307

Bartelmann M., Schneider P., 2001, Phys. Rep, 340, 291

Becker M. R. et al., 2016, PRD, 94, 022002

Brouwer M. M. et al., 2016, MNRAS, 462, 4451

Bunn E. F., Zaldarriaga M., Tegmark M., de Oliveira-Costa A., 2003, PRD, 67,023501

Castro P. G., Heavens A. F., Kitching T. D., 2005, PRD, 72, 023516

Chang C. et al., 2015, Phys. Rev. Lett., 115, 051301

Chang C. et al., 2018, MNRAS, 475, 3165

Clowe D., Bradač M., Gonzalez A. H., Markevitch M., Randall S. W., Jones C., Zaritsky D., 2006, ApJ, 648, L109

Coles P., Chiang L. Y., 2000, Nature, 406, 376

Crittenden R. G., Natarajan P., Pen U. L., Theuns T., 2001, ApJ, 559, 552

Crittenden R. G., Natarajan P., Pen U. L., Theuns T., 2002, ApJ, 568, 20

de Jong J. T. A., Verdoes Kleijn G. A., Kuijken K. H., Valentijn E. A., 2013, Exp. Astron., 35, 25

Flaugher B. et al., 2015, AJ, 150, 150

Goldberg J. N., Macfarlane A. J., Newman E. T., Rohrlich F., Sudarshan E. C. G., 1967, J. Math. Phys., 8, 2155

Górski K. M., Hivon E., Banday A. J., Wandelt B. D., Hansen F. K., Reinecke M., Bartelmann M., 2005, ApJ, 622, 759

Green D. A., 2011, Bull. Astron. Soc. India, 39, 289

Heavens A., 2009, Nucl. Phys. B, 194, 76

Heymans C. et al., 2012, MNRAS, 427, 146

Hirata C. M., Seljak U., 2004, PRD, 70, 063526

Hobson M., Jones A., Lasenby A., 1998, MNRAS, 309, 125

Jeffrey N. et al., 2021, MNRAS, 505, 4626

Jullo E., Kneib J. P., Limousin M., Elíasdóttir Á., Marshall P. J., Verdugo T., 2007, New J. Phys., 9, 447

Jullo E., Pires S., Jauzac M., Kneib J. P., 2014, MNRAS, 437, 3969

Kaiser N., Squires G., 1993, ApJ, 404, 441

Kamionkowski M., Kosowsky A., Stebbins A., 1997, PRD, D55, 7368

Kaufman J. P., Keating B. G., Johnson B. R., 2016, MNRAS, 455, 1981

Kilbinger M., 2015, Rep. Prog. Phys., 78, 086901

Kirk D. et al., 2015, Space Sci. Rev., 193, 139

Kitching T. D., Alsing J., Heavens A. F., Jimenez R., McEwen J. D., Verde L., 2016,MNRAS, 469, 2737

Kratochvil J. M., Lim E. A., Wang S., Haiman Z., May M., Huffenberger K., 2012, PRD, 85, 103513

Lanusse F., Starck J. L., Leonard A., Pires S., 2016, A\&A, 591, A2

Laureijs R. et al., 2011, preprint (arXiv:1110.3193)

Leistedt B., McEwen J. D., Vandergheynst P., Wiaux Y., 2013, A\&A, 558, 1

Leistedt B., McEwen J. D., Büttner M., Peiris H. V., 2017, MNRAS, 466, 3728

Leonard A., Dupé F. X., Starck J. L., 2012, A\&A, 539, A85

Leonard A., Lanusse F., Starck J. L., 2014, MNRAS, 440, 1281

Lin C. A., Kilbinger M., 2015a, A\&A, 576, A24

Lin C. A., Kilbinger M., 2015b, A\&A, 583, A70

Lin C. A., Kilbinger M., Pires S., 2016, A\&A, 593, A88

${ }^{14}$ https://github.com/astro-informatics/massmappy 
Liu J., Hill J. C., 2015, PRD, 92, 063517

LSST Science Collaboration et al., 2009, preprint (arXiv:0912.0201)

Marinucci D., Peccati G., 2011, Random Fields on the Sphere: Representation, Limit Theorem and Cosmological Applications, Cambridge Univ. Press, Cambridge

Massey R. et al., 2004, AJ, 127, 3089

Massey R. et al., 2007, Nature, 445, 286

Massey R. et al., 2015, MNRAS, 449, 3393

McEwen J. D., Wiaux Y., 2011, IEEE TSP, 59, 5876

McEwen J. D., Hobson M. P., Lasenby A. N., Mortlock D. J., 2005, MNRAS, 359,1583

McEwen J. D., Leistedt B., Büttner M., Peiris H. V., Wiaux Y., 2015, preprint (arXiv:1509.06749)

Mediavilla E., Muñoz J. A., Garzón F., Mahoney T. J., 2016, Astrophysical Applications of Gravitational Lensing, Cambridge Univ. Press, Cambridge

Munshi D., Valageas P., van Waerbeke L., Heavens A., 2008, Phys. Rep, 462, 67

Munshi D., Kitching T., Heavens A., Coles P., 2011, MNRAS, 416, 1629

Munshi D., van Waerbeke L., Smidt J., Coles P., 2012, MNRAS, 419, 536

Newman E. T., Penrose R., 1966, J. Math. Phys., 7, 863

Peel A., Lin C. A., Lanusse F., Leonard A., Starck J. L., Kilbinger M., 2016, A\&A, 599, 12

Petri A., Haiman Z., Hui L., May M., Kratochvil J. M., 2013, PRD, 88, 123002

Pichon C., Thiébaut E., Prunet S., Benabed K., Colombi S., Sousbie T., Teyssier R., 2010, MNRAS, 401, 705

Plaszczynski S., Lavabre A., Perotto L., Starck J. L., 2012, A\&A, 544, A27

Price M. A., Cai X., McEwen J. D., Pereyra M., Kitching T. D., LSST Dark Energy Science Collaboration, 2020, MNRAS, 492, 394

Price M. A., McEwen J. D., Cai X., Kitching T. D., Wallis C. G. R., 2021, MNRAS, 506, 3678

Price M. A., McEwen J. D., Pratley L., Kitching T. D., 2021, MNRAS, 500, 5436

Schneider P., 2005, ArXiv Astrophysics e-prints preprint( astro-ph/0509252)

Scoville N. et al., 2007, ApJ S., 172, 1

Seitz C., Schneider P., 1995, A\&A, 297, 287

Simon P., 2013, A\&A, 560, A33

Simon P., Taylor A. N., Hartlap J., 2009, MNRAS, 399, 48

Spergel D. et al., 2015, Wide-field infrarred survey telescope-astrophysics focused telescope assets WFIRST-AFTA 2015 report, preprint (arXiv:1503.03757)

Szepietowski R. M., Bacon D. J., Dietrich J. P., Busha M., Wechsler R., Melchior P., 2014, MNRAS, 440, 2191

Taylor A. N., 2001, preprint( astro-ph/0111605)

Taylor A. N. et al., 2004, MNRAS, 353, 1176

Vallis Z., Wallis C. G., Kitching T. D., 2018, Astron. Comput., 24, 84

Van Waerbeke L. et al., 2013, MNRAS, 433, 3373

VanderPlas J. T., Connolly A. J., Jain B., Jarvis M., 2011, ApJ, 727, 118

Varshalovich D. A., Moskalev A. N., Khersonskii V. K., 1989, Quantum theory of angular momentum, World Scientific, Singapore

Vielva P., Martínez-González E., Barreiro R. B., Sanz J. L., Cayón L., 2004, ApJ, 609, 22

Vikram V. et al., 2015, PRD, 92, 022006

Wallis C. G. R., Wiaux Y., McEwen J. D., 2017, IEEE Transactions on Image Processing, 26, 5176

Zaldarriaga M., Seljak U., 1997, PRD, 55, 1830

Zuntz J. et al., 2015, Astron. Comput., 12, 45

\section{APPENDIX A: EQUIVALENCE OF DIFFERENT REPRESENTATIONS OF SPHERICAL MASS-MAPPING INVERSE PROBLEM}

The equivalence of the harmonic and integral expressions, equations (24) and (28), respectively, connecting the observable cosmic shear field to the convergence field can also be shown by considering the harmonic representation of the integral expression. Consider the integral representation, decomposing the kernel and convergence field into their harmonic expansions

$$
\begin{aligned}
& 2 \gamma(\omega)=\int_{\mathbb{S}^{2}} \mathrm{~d} \Omega\left(\omega^{\prime}\right)\left(\mathcal{R}_{\omega^{\prime}} 2 \mathcal{K}\right)(\omega) 0 \kappa\left(\omega^{\prime}\right) \\
& =\int_{\mathbb{S}^{2}} \mathrm{~d} \Omega\left(\omega^{\prime}\right) \sum_{\ell m} 2 \mathcal{K}_{\ell m}\left(\mathcal{R}_{\omega^{\prime}} 2 Y_{\ell m}\right)(\omega) \\
& \sum_{\ell^{\prime} m^{\prime}} 0 \hat{\kappa}_{\ell^{\prime} m^{\prime} 0} Y_{\ell^{\prime} m^{\prime}}\left(\omega^{\prime}\right) .
\end{aligned}
$$

The rotation of the spin spherical harmonic in the above expression is given by

$$
\begin{aligned}
& \left(\mathcal{R}_{\omega^{\prime}} 2 Y_{\ell 0}\right)(\omega)=\sum_{n} D_{n 0}^{\ell}\left(\omega^{\prime}\right) 2 Y_{\ell n}(\omega) \\
& =\sqrt{\frac{4 \pi}{2 \ell+1} \sum_{n} 0 Y_{\ell n}^{*}\left(\omega^{\prime}\right) 2 Y_{\ell n}(\omega),}
\end{aligned}
$$

where it is necessary to only consider $m=0$ due to the Kronecker delta term $\delta_{m 0}$ appearing in $2 \mathcal{K}_{\ell m}$, as shown in equation (30), and noting equations (10) and (11). Equation (A2) can then be written as

$2 \gamma(\omega)=\sum_{\ell n} \frac{-1}{\ell(\ell+1)} \sqrt{\frac{(\ell+2) !}{(\ell-2) !}} 0 \hat{\kappa}_{\ell n} 2 Y_{\ell n}(\omega)$,

where we have noted the orthogonality of the spherical harmonics, i.e. $\left\langle Y_{\ell m}, Y_{\ell^{\prime} m^{\prime}}\right\rangle=\delta_{\ell \ell^{\prime}} \delta_{m m^{\prime}}$. The resulting harmonic representation of equation (28) is, thus, identical to equation (24), as expected.

\section{APPENDIX B: PROJECTIONS}

In this appendix, we outline the details of each projection considered. We first define each projection and describe its properties. Each projection has different beneficial properties, for example whether the projection is equal-area, has appropriate boundary conditions or conformal. Conformal projections conserve local angles and are often used for geographical maps. We also describe the distance metric we use for each projection to define the opening angle of the patch of sky seen by an experiment, i.e. the angle considered in Fig. 4. We then detail how to calculate the local rotation angles required when projecting spin fields, such as shear (without this rotation $E$ - and $B$-modes will be misinterpreted) and finally illustrate the impact of neglecting this local rotation on DES SV data.

\section{B1 Projection definitions}

We consider two general types of projection: equatorial and polar projections. Equatorial projections are defined relative to the equator, while polar projections are defined relative to a pole. The precise definitions of the different equatorial and polar projections are given in the following subsections. The equatorial projections considered include: the sinusoidal projection, which is a simple equal area projection that was used by the DES collaboration; the Mercator projection that is a conformal projection, often used in geographical maps as it preserves local angles; and a simple cylindrical projection. The polar projections considered include: the orthographic projection, which is a simple vertical projection from the sphere to a tangent plane; the Gnomonic projection that has the useful property that the local rotations are trivial to calculate; and the stereographic projection that is another conformal projection. 


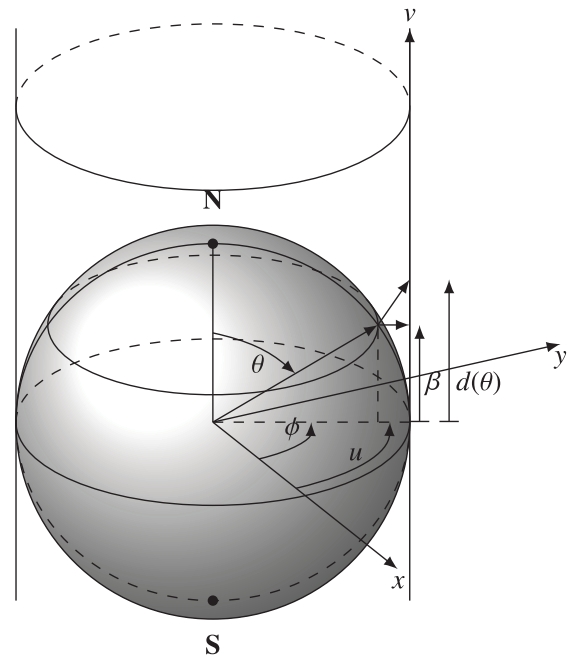

Figure B1. Diagram to describe graphically the equatorial projections, including the sinusoidal, Mercator, and the simple cylindrical projections. These can all be seen as types of cylindrical projections since the sphere is projected onto a cylinder wrapped round the sphere. The $u$ variable simply describes how far round the cylinder a point is and is therefore give by $\phi$ (up to some arbitrary shift), except in the sinusoidal case where the $u$ variable is contracted away from the equator to ensure the projection is equal-area. The $v$ variable can vary between projections and can be specified by various functions $d(\theta)$. In the Mercator projection, this function is chosen to ensure the projection is conformal. In the sinusoidal and simple cylindrical projections, this function is simply $d(\theta)=\beta=\pi / 2-\theta$.

\section{B1.1 Equatorial projections}

Fig. B1 shows graphically how the equatorial projections can be viewed as a projection on to a cylinder wrapped round the sphere. Each projection is defined by the relation between the spherical coordinates $(\theta, \phi)$ and the planar coordinates $(u, v)$.

The sinusoidal projection (used by the DES collaboration) is defined by

$u=(\phi-\pi) \sin (\theta)$,

$v=\theta$.

This projection results in minimal distortion in the central region $(\theta=$ $\pi / 2, \phi=\pi$ ). Moving away from this point in any direction increases the distortion but particularly in a diagonal direction (specifically along the lines $y=x$ or $y=-x$ ). We define the distance metric for this projection by

$\Theta=\sqrt{(\theta-\pi / 2)^{2}+(\phi-\pi)^{2}}$.

The sinusoidal projection has the useful property of being equalarea. It is simpler to define than the Mollweide projection, also an equal-area projection, which is commonly used for plotting in the cosmological community.

The Mercator projection is commonly used for geographical maps and is defined by

$u=\phi-\pi$,

$v=\ln [\tan (\pi / 2-\theta / 2)]$.

This projection has the useful property of being conformal, meaning that local angles on the sphere will not be distorted. The projection introduces minimal distortion at the equator, while the projected image is stretched and distorted as one moves towards the pole. Since the poles themselves are at infinity the projection cannot completely cover the full sky in practice. The projection is a cylindrical projection

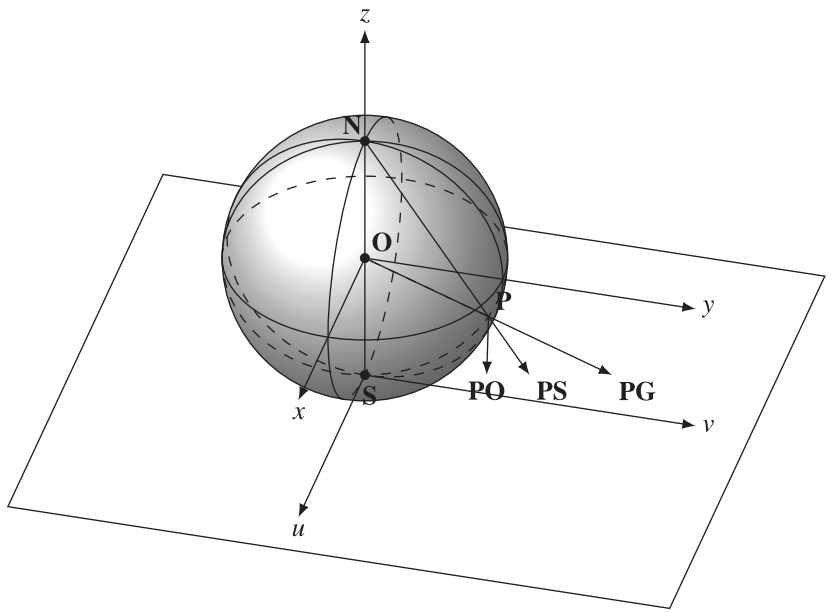

Figure B2. Diagram to describe graphically the polar projections, including the orthographic, stereographic, and gnomic projections. In these projections, a point on the sphere is projected to the tangent plane at a pole (here chosen to be the South Pole). For projections around the North or South Pole, the angle $\phi$ is simply taken as the polar coordinate $\varphi$ in the planar space. The radial coordinate $\varrho$ is a function of the angle between the point and the pole whose tangent plane is considered ( $\pi-\theta$ for the South Pole). The orthographic projection is a vertical projection, giving $\varrho=\sin (\pi-\theta)$ for the tangent plane at the South Pole. The gnomic projection casts a ray from the origin to the point on the sphere and through to the tangent plane, giving $\varrho=\tan (\pi-\theta)$ for the tangent plane at the South pole. Finally, the stereographic projection casts a ray from the opposite pole to the point on the sphere and through to the tangent plane, giving $\varrho=2 \tan [(\pi-\theta) / 2]$ for the tangent plane at the South Pole. In the diagram, the point $\mathrm{P}$ is projected to PO, PS, and PG by the orthographic, stereographic, and gnomic projections, respectively.

and therefore has the correct boundary conditions in the $u$ direction. The metric used to define the angular distance from the undistorted region is simply given by

$\Theta=|\theta-\pi / 2|$.

The final equatorial projection we consider is the simple cylindrical projection defined by

$u=\phi-\pi$

$v=\theta-\pi / 2$.

There are no particular properties to inspire us to propose this projection over the more sophisticated cylindrical projection of the Mercator projection. Its attractiveness is in its simplicity and the ability to map the entire sphere on one plane. The distortions increase away from the equator leading to the same distance metric as the Mercator projection, i.e. equation (B4).

\section{B1.2 Polar projections}

Fig. B2 shows a graphical representation of the polar projections, where again the spherical coordinates $(\theta, \phi)$ are projected on to the planar coordinates $(u, v)$. It is most straightforward to define these projections using polar coordinates on the plane $(\varrho, \varphi)$, which are related to the Cartesian coordinates by

$u=\varrho \cos (\varphi)$,
$v=\varrho \sin (\varphi)$.

In each of the polar projections, we simply have that $\varphi=\phi$. The projections differ in the way $\theta$ is mapped to $\varrho$, where each projection 
has its own mapping function $f$, i.e.

$\varrho=f(\theta)$.

It is a common feature of these projections that the entire sphere cannot be projected to a single plane in practice (since in many cases the opposite pole is mapped to the point at infinity). In that case, we often project around the South Pole as well as the North Pole and consider $\varrho=f(\pi-\theta)$. We define the distance metric for these projections to be

$\Theta=\theta$.

The orthographic projection is defined by

$\varrho=\sin (\theta)$.

For this projection, a point on the sphere is mapped vertically from the sphere to the tangent plane at the North Pole. As a result, the whole sphere cannot be projected on to one plane in practice and one must project each hemisphere on to a different plane.

We also consider the Gnomonic projection defined by casting a ray from the centre of the sphere to the point considered and then through to the tangent plane at the North Pole. The Gnomonic projection is therefore defined by

$\varrho=\tan (\theta)$.

For this projection, the whole sphere again cannot be projected on to one plane in practice since the equator is projected to infinity. One must again project the sphere into a number of regions, for example considering each hemisphere separately.

The final projection we consider is the stereographic projection. This is defined by casting a ray from the South Pole to the point considered on the sphere and then through to the tangent plane at the North Pole. The resulting projection is defined by

$\varrho=2 \tan (\theta / 2)$.

We can project almost all of the sphere with this projection, except near the South Pole, as the South Pole is mapped to infinity. This projection is conformal, preserving local angles.

\section{B2 Rotation angles}

Spin fields on the sphere have local directions defined relative to the North pole, whereas on the plane the spin fields have their spin defined relative to some universal direction (usually the 'top' of the planar map). We define this direction on the plane by $\hat{\boldsymbol{v}}$, the unit vector in the $v$ direction. On projection, the spin field must be rotated from its original coordinate frame on the sphere to the new coordinate frame on the plane. Here, we describe how to calculate this local rotation angle.

When we project from the sphere to the plane, it is common to rotate our coordinate system before we project. This is done in order to centre the region of interest so that distortions due to the projection are minimized at this point. We therefore need to define a number of coordinate systems, including the original sphere, the rotated sphere, and the plane. First, consider a field defined on the original sphere with spherical coordinates $\left(\theta^{\prime}, \phi^{\prime}\right)$ and corresponding Cartesian coordinates $\left(x^{\prime}, y^{\prime}, z^{\prime}\right)$. Consider then the rotated field, where the spherical coordinates of the rotated sphere are $(\theta, \phi)$, with corresponding Cartesian coordinates $(x, y, z)$. We define the rotation relating the primed frame to the unprimed frame by $\mathcal{R}_{\rho}$, with corresponding $3 \mathrm{D}$ rotation matrix $\mathbf{R}$. From the rotated sphere, the field is then projected on to the plane defined by Cartesian coordinates $(u, v)$ and polar coordinates $(\varrho, \varphi)$.
We need to find the angle between $\hat{v}$ and the projected direction of the North pole of the original sphere. To do this, we consider an infinitesimal step North on the sphere and then find the infinitesimal step this makes on the plane $(\mathrm{d} u, \mathrm{~d} v)$. The rotation angle $\psi$ required is then the angle between the $\hat{v}$ direction and the projected North direction.

\section{B2.1 Equatorial projections}

The first step is to construct a vector that is an infinitesimal step North in the original space. This vector is given by

$\mathbf{d} \boldsymbol{x}^{\prime}=\left(\begin{array}{l}0 \\ 0 \\ 1\end{array}\right) \mathrm{d} \epsilon$,

where $\mathrm{d} \epsilon$ is an infinitesimal element of the real line. When this infinitesimal element is projected on to the sphere at any point, it always points North (with the exception of the poles). Moving in this direction, thus, yields a vector that is further North but is not normalized to lie on the unit sphere. The normalization of the vector is unimportant as later on in this proof we require the direction of this vector only and not its length. In the unprimed frame, this infinitesimal step is given by

$$
\begin{gathered}
\mathbf{d} \boldsymbol{x}=\mathbf{R d} \boldsymbol{x}^{\prime}, \\
\left(\begin{array}{l}
\mathrm{d} x \\
\mathrm{~d} y \\
\mathrm{~d} z
\end{array}\right)=\left(\begin{array}{l}
\mathbf{R}_{1,3} \\
\mathbf{R}_{2,3} \\
\mathbf{R}_{3,3}
\end{array}\right) \mathrm{d} \epsilon .
\end{gathered}
$$

Now we apply the chain rule twice to calculate the projected infinitesimal step in the plane $(\mathrm{d} u, \mathrm{~d} v)$. First, we note the relation between $(x, y, z)$ and $(\theta, \phi)$ of

$$
\begin{aligned}
& \theta=\arctan \left(\frac{\sqrt{x^{2}+y^{2}}}{z}\right), \\
& \phi=\arctan \left(\frac{y}{x}\right),
\end{aligned}
$$

where the normalization of the vector is unimportant, ensuring the definition of $\mathbf{d} \boldsymbol{x}^{\prime}$ is acceptable. Applying the chain rule, we have

$$
\begin{aligned}
\mathrm{d} \theta & =\cos (\theta)[\cos (\phi) \mathrm{d} x+\sin (\phi) \mathrm{d} y-\tan (\theta) \mathrm{d} z], \\
\mathrm{d} \phi & =\csc (\theta)[-\sin (\phi) \mathrm{d} x+\cos (\phi) \mathrm{d} y],
\end{aligned}
$$

where a unit vector is assumed without loss of generality. We now generalize the equatorial projections as

$$
\begin{aligned}
& u=g(\theta, \phi), \\
& v=h(\theta, \phi) .
\end{aligned}
$$

We then apply the chain rule again to give

$$
\begin{aligned}
& \mathrm{d} u=\frac{\partial g}{\partial \theta} \mathrm{d} \theta+\frac{\partial g}{\partial \phi} \mathrm{d} \phi, \\
& \mathrm{d} v=\frac{\partial h}{\partial \theta} \mathrm{d} \theta+\frac{\partial h}{\partial \phi} \mathrm{d} \phi,
\end{aligned}
$$

from which the rotation angle $\psi$ can be calculated by

$\psi=-\arctan \left(\frac{\mathrm{d} u}{\mathrm{~d} v}\right)$

After substituting all the terms from the above expressions into equation (B18), $\mathrm{d} \epsilon$ cancels out and the limit $\mathrm{d} \epsilon \rightarrow 0$ follows trivially. 


\section{B2.2 Polar projections}

For polar projections, the calculation begins in the same way as for equatorial projections, up until equation (B15). Then, we apply the chain rule giving

$$
\begin{aligned}
\mathrm{d} \varrho & =\frac{\mathrm{d} f}{\mathrm{~d} \theta} \mathrm{d} \theta, \\
\mathrm{d} \varphi & =\mathrm{d} \phi .
\end{aligned}
$$

Applying the chain rule again to the relation between $(u, v)$ and $(\varrho, \varphi)$ of equation (B6), we have

$$
\begin{aligned}
\mathrm{d} u & =\cos (\varphi) \mathrm{d} \varrho-\varrho \sin (\varphi) \mathrm{d} \varphi, \\
\mathrm{d} v & =\sin (\varphi) \mathrm{d} \varrho+\varrho \cos (\varphi) \mathrm{d} \varphi .
\end{aligned}
$$

We then compute the local rotation angle $\psi$ in the same manner as above, i.e. by equation (B18). It is possible to show from this result the special property of the Gnomonic projection: when there is no rotation and $f(\theta)=\tan (\theta)$, as is the case for the Gnomonic projection, the rotation angle is zero everywhere.

\section{B3 Application to DES SV data}

Here, we demonstrate the importance of applying this rotation in practice, using DES SV data. As far as we are aware, applying these local rotations is not standard practice. We consider the sinusoidal projection also used by the DES collaboration. However, here we do not apply the necessary rotations to the galaxy shapes (as we did in the main body of the article). Figs B3(a) and B3(b) show the results when no rotation is applied and Figs B3(c) and B3(d) show the error introduced by not applying the local rotations, i.e. the differences with the maps shown in Figs 6(a) and (b). While the effect is not large for DES SV data, it is not insignificant. Furthermore, if considering planar mass-mapping techniques for larger survey coverages this effect becomes increasingly important. 


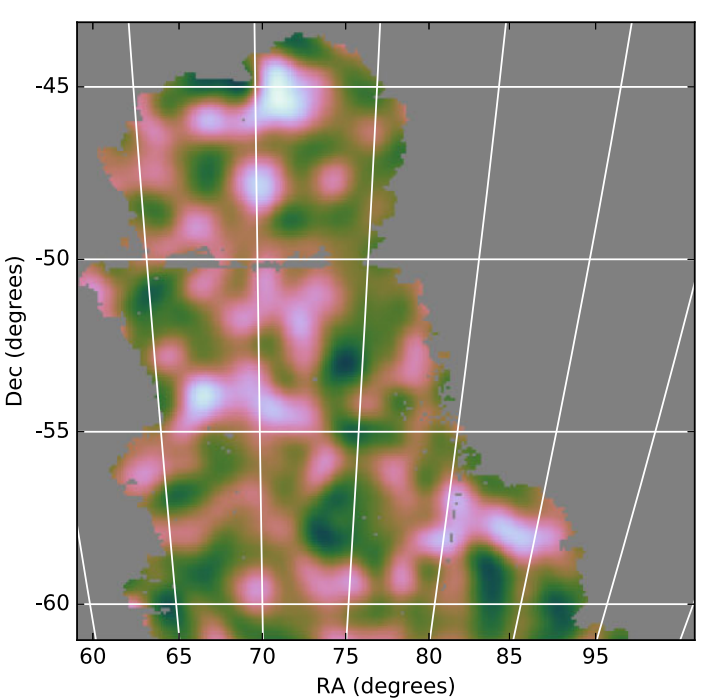

(a) $\kappa^{\mathrm{KS}} E$-mode with no local rotations

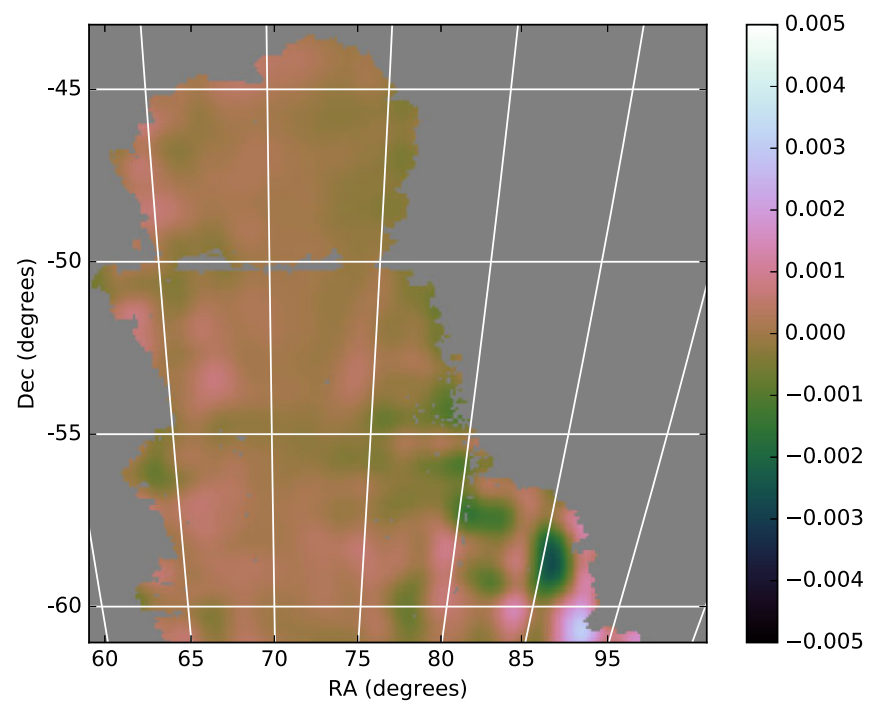

(c) Difference between $\kappa^{\mathrm{KS}} E$-mode with and without local rotations

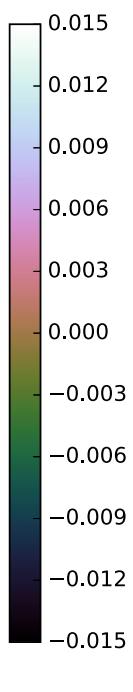

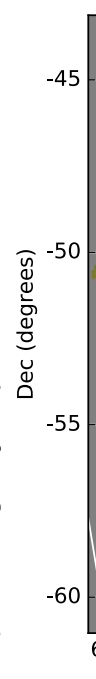

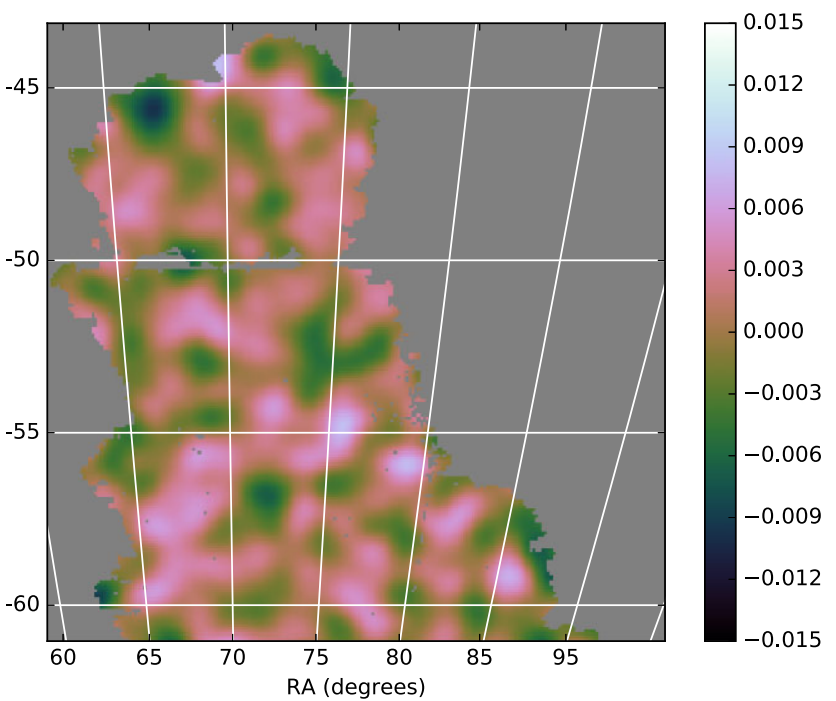

(b) $\kappa^{\mathrm{KS}} B$-mode with no local rotations

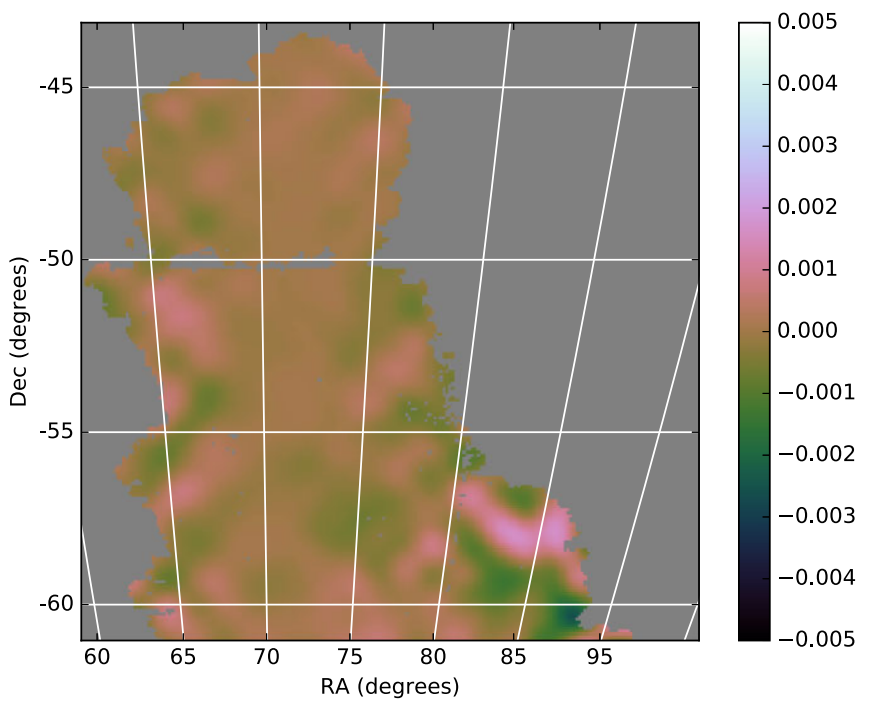

(d) Difference between $\kappa^{\mathrm{KS}} B$-mode with and without local rotations

Figure B3. Plot to show the importance of applying the local rotations to real data when performing projections. We project the DES SV data using the sinusoidal projection considered by the DES collaboration. However, in this case, we do not apply the necessary rotations to the galaxy shapes. Panels (a) and (b) show the results when no rotation is applied, while panels (c) and (d) show the error introduced by not applying the local rotations, i.e. the differences with the maps shown in Figs 6(a) and (b).

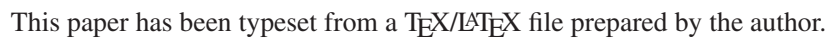

